\title{
Quantum control theory and applications: A survey *广
}

\author{
Daoyi Dong $\stackrel{\ddagger}{\ddagger} \quad$ Ian R. Petersen ${ }^{\S}$
}

9 January, 2011

\begin{abstract}
This paper presents a survey on quantum control theory and applications from a control systems perspective. Some of the basic concepts and main developments (including open-loop control and closed-loop control) in quantum control theory are reviewed. In the area of open-loop quantum control, the paper surveys the notion of controllability for quantum systems and presents several control design strategies including optimal control, Lyapunov-based methodologies, variable structure control and quantum incoherent control. In the area of closedloop quantum control, the paper reviews closed-loop learning control and several important issues related to quantum feedback control including quantum filtering, feedback stabilization, LQG control and robust quantum control.
\end{abstract}

Key words: quantum control, controllability, coherent control, incoherent control, feedback control, robust control.

\section{Introduction}

Quantum control theory is a rapidly evolving research area, which has developed over the last three decades [1-9]. Controlling quantum phenomena has been an implicit goal of much quantum physics and chemistry research since the establishment of quantum mechanics [5], [6]. One of the main goals in quantum control theory is to establish a firm theoretical footing and develop a series of systematic methods for the active manipulation and control of quantum systems [7]. This goal is nontrivial since microscopic quantum systems have many unique characteristics (e.g., entanglement and coherence) which do not occur in classical mechanical systems and the dynamics of quantum systems must be described by quantum theory. Quantum control theory has already attained significant successes in physical chemistry [9]-[13], atomic and molecular physics [6], [14] and quantum optics [15], [16], and has also contributed to the

\footnotetext{
${ }^{*}$ This paper has been published by IET Control Theory \& Applications, vol. 4, no. 12, pp.2651-2671.

${ }^{\dagger}$ This work was supported by the Australian Research Council and was supported in part by the National Natural Science Foundation of China under Grant No. 60703083.

${ }^{\ddagger}$ D. Dong is with the School of Engineering and Information Technology, University of New South Wales at the Australian Defence Force Academy, Canberra, ACT 2600, Australia (email: daoyidong@gmail.com).

${ }^{\S}$ I. R. Petersen is with the School of Engineering and Information Technology, University of New South Wales at the Australian Defence Force Academy, Canberra, ACT 2600, Australia (email: i.r.petersen@gmail.com).
} 
understanding of fundamental aspects of quantum mechanics [17]. In recent years, the development of the general principles of quantum control theory has been recognized as an essential requirement for the future application of quantum technologies [18]. Quantum control theory is drawing wide attention from different communities in the areas of physics, chemistry, control theory, applied mathematics and quantum information, and has become a regular topic in international conferences such as the IEEE Conference on Decision and Control. An international workshop on "Principles and Applications for Control of Quantum Systems (PRACQSYS)" has also been sponsored since 2004 to discuss recent developments in this area [7. This paper will present the basic concepts and main developments of quantum control theory from a control systems perspective.

In much of quantum control theory, the controllability of quantum systems is a fundamental issue. This issue concerns whether one can drive a quantum system to a desired state [3. This problem has practical importance since it has a close connection with the universality of quantum computation [19] and the possibility of achieving atomic or molecular scale transformations [20, [21. Different notions of controllability, such as pure state controllability, complete controllability, eigenstate controllability and kinematic controllability, have been proposed [20]-[26]. A common research focus is on finite dimensional (finite level) quantum systems for which the controllability criteria may be expressed in terms of the structure and rank of corresponding Lie groups and Lie algebras [25]. This method allows for an easy mathematical treatment of the problem for the case of closed quantum systems (i.e., isolated systems considered without external influences) and in some cases, methods from classical (non-quantum) control theory can be directly applied. However, the relevant criteria may be computationally difficult when the dimension of the quantum system to be controlled is large. Therefore, a controllability method based on graph theory has been proposed, and the corresponding controllability criterion is easier to verify [27], 28]. Compared with finite dimensional closed quantum systems, the controllability of infinite dimensional quantum systems or open quantum systems (i.e., systems considered interacting with the environment; e.g., see [29]) is more difficult and only a few results have been obtained [21, [26], [30].

For a controllable quantum system, it is desirable to develop a good control strategy to accomplish the required control tasks on the quantum system [9]. The coherent control strategy is a widely used method where one manipulates the states of a quantum system by applying semiclassical potentials in a fashion that preserves quantum coherence [17] (a wave-like property of quantum systems allowing for constructive and destructive interference, e.g., see [5]). An early paradigm of quantum control was openloop coherent control [5], which has successfully been used in the quantum control of chemical reactions [31], 32]. Rigorous optimal control theory has been successfully applied to the design of open-loop coherent control strategies in order to find the best way of achieving given quantum control objectives in physical chemistry [12, [33, [34. Recently, time-optimal control problems for spin systems have been solved to achieve specified control objectives in minimum time [35], [36, 33]. Optimal control techniques have also been successfully applied to multidimensional nuclear magnetic resonance (NMR) experiments to improve the sensitivity of these systems in the presence of relaxation [38, 39], 40]. Another useful tool in open-loop quantum control is 
the Lyapunov-based control design approach [41, [42]. In classical control, the Lyapunov method determines the control input as a function of the system state. Hence, it is a feedback control design method. In quantum control, it is difficult to acquire information about quantum states without destroying them. Hence, the Lyapunov-based control methodology is used to first construct an artificial closed-loop controller and then an open-loop control law is obtained by simulation of the artificial closed-loop system. In coherent control, the control operations consist of unitary transformations. However, some quantum systems may not be controllable using only coherent controls. For such uncontrollable quantum systems, it may be possible to enhance the capabilities of quantum control by introducing new control strategies such as variable structure control and incoherent control (i.e., one is allowed to destroy coherence of the quantum systems during the control process). For example, a variable structure control method has been introduced in [43, 44] to enhance the capabilities of quantum control for some specific systems which are not controllable. Incoherent control enables the effects of quantum measurements and incoherent environments to be combined with unitary transformations to complete quantum control tasks and enhance the performance of quantum control systems [45]-[55]. Incoherent control strategies have also been recognized as important techniques to assist coherent control strategies in quantum control systems.

Although open-loop strategies using coherent or incoherent control approaches, optimal control techniques and Lyapunov methods, have achieved important successes, their applications have many limitations. In classical control, closed-loop methods have many advantages over open-loop methods, such as robustness and reliability. For open quantum systems, coupling with uncontrollable environments makes the introduction of noises and uncertainties unavoidable [56]. A requirement for robustness in the presence of uncertainties and noises has been recognized as one of the key aspects in developing practical quantum technologies [7], [57. A natural solution to this problem is to develop closed-loop quantum control approaches. Two paradigms for closed-loop control have been proposed: closed-loop learning control and quantum feedback control [9]. Closedloop learning control involves a closed-loop operation where each cycle of the loop is executed with a new sample, and this approach has achieved great success in controlling quantum phenomena in chemical reactions [9, [58. Feedback is an essential concept in classical control theory, where information about the state variables obtained from direct measurements or state estimation is fed back to the system through a controller to achieve desired performance. Quantum feedback control has been used to improve the system performance in different tasks, including the control of squeezed states (a typical class of nonclassical states, e.g., see [59]) and entangled states (see Section 2.1), state reduction and quantum error correction [15], 60]-69]. The success of feedback control is usually dependent on the acquisition of suitable feedback information. This problem becomes more complex and subtle in quantum control since quantum measurements unavoidably affect the states of measured systems. Hence, research aimed at establishing a general framework for quantum feedback is accelerating the development of some related areas including quantum filtering theory [70], 71], [72], quantum parameter estimation [73], 74], [75], quantum system identification [76], 77], 78] and 
quantum robust control [57], [79].

This paper will survey the development of open-loop and closed-loop methods in quantum control theory from a control systems perspective. We will also briefly discuss some applications of quantum control theory to quantum information technology [80]. Many successful applications of quantum control in chemistry and physics are beyond the scope of this paper and, for these topics, readers can refer to several excellent books and review articles in the areas of chemical reactions [5, [9, [11, [12], [13, [14, [31, [32, atomic physics [6], NMR [7, 81] and quantum optics [82]. The remainder of this paper is organized as follows. Section 2 briefly introduces quantum states, quantum measurements and several quantum control models. In Section 3 , the controllability of quantum systems and several open-loop control strategies, including optimal control, Lyapunov methods, incoherent control and variable structure control, are reviewed. Section 4 presents some results on closed-loop control of quantum systems. In particular, in this section, closed-loop learning control is briefly mentioned and some specific aspects of quantum feedback control are presented. Conclusions and perspectives are given in Section 5 .

\section{Prerequisites}

In quantum control theory, the systems to be controlled are quantum systems whose dynamics are governed by the laws of quantum mechanics. Quantum mechanics provides a mathematical framework for describing the states and evolution of quantum systems. In this section, we briefly introduce several related concepts for developing quantum control theory, such as quantum states, quantum measurements and quantum control models. A comprehensive introduction to quantum mechanics can be found in many excellent textbooks such as [83], 84].

\subsection{Quantum states}

In quantum mechanics, the state of a closed quantum system can be represented by a unit vector $|\psi\rangle$ (this notation is known as the Dirac representation [83] and the state $|\psi\rangle$ is also called wavefunction) in a complex Hilbert space $\mathcal{H}$. It is worth mentioning that the "state" in quantum mechanics is different from that in classical systems. For a classical system, the state usually describes some real physical properties such as position and momentum, which are generally observable. However, a quantum state cannot be directly observed and also does not directly correspond to physical quantities of the quantum system. Since the global phase of a quantum state has no observable physical effect, we often say that the vectors $|\psi\rangle$ and $e^{i \alpha}|\psi\rangle$ (where $i=\sqrt{-1}$ and $\alpha \in \mathbb{R}$ ) describe the same physical state. A quantum state which can be represented with a unit vector $|\psi\rangle$ is called a pure state. For example, in quantum information theory, information is coded using two-state (two-level) quantum systems (called qubits) and the state $|\psi\rangle$ of a qubit can be written as

$$
|\psi\rangle=\cos \frac{\theta}{2}|0\rangle+e^{i \varphi} \sin \frac{\theta}{2}|1\rangle
$$


where $\theta \in[0, \pi], \varphi \in[0,2 \pi]$. Then $|0\rangle$ and $|1\rangle$ correspond to the states zero and one for a classical bit [80], [85].

In practical applications, the quantum systems to be controlled are usually not simple closed systems. They may be quantum ensembles or open quantum systems and their states cannot be written in the form of unit vectors. In this case, it is necessary to introduce the density operator or density matrix $\rho: \mathcal{H} \rightarrow \mathcal{H}$ to describe quantum states of quantum ensembles or open quantum systems. A density operator $\rho$ is positive and has trace equal to one. Suppose that a quantum system is in an ensemble $\left\{p_{j},\left|\psi_{j}\right\rangle\right\}$ of pure states; i.e., in a mixture of a number of pure states $\left|\psi_{j}\right\rangle$ with respective probabilities $p_{j}$. The density matrix for the system is defined as follows 80]:

$$
\rho \equiv \sum_{j} p_{j}\left|\psi_{j}\right\rangle\left\langle\psi_{j}\right|
$$

where $\left\langle\psi_{j}\right|=\left(\left|\psi_{j}\right\rangle\right)^{\dagger}$ and $\sum_{j} p_{j}=1$. Here, the operation $(\cdot)^{\dagger}$ refers to the adjoint. For a pure state $|\psi\rangle, \rho=|\psi\rangle\langle\psi|$ and $\operatorname{tr}\left(\rho^{2}\right)=1$. If the state $\rho$ of a quantum system satisfies $\operatorname{tr}\left(\rho^{2}\right)<1$, we call the quantum state a mixed state.

A composite quantum system (assumed to be made up of two subsystems $A$ and $B$ ) is defined on a Hilbert space $\mathcal{H}=\mathcal{H}_{A} \otimes \mathcal{H}_{B}$, which is the tensor product of the Hilbert spaces $\mathcal{H}_{A}$ and $\mathcal{H}_{B}$ on which the subsystems $A$ and $B$ are defined. For the composite quantum system, its state $\rho_{A B}$ can be described by the tensor product of the states of its subsystems; i.e., $\rho_{A B}=\rho_{A} \otimes \rho_{B}$ (e.g., see [80]).

Considering any bipartite pure state $|\psi\rangle_{A B}$, if it can be written as a tensor product of pure states $|\varphi\rangle_{A} \in \mathcal{H}_{A}$ and $|\chi\rangle_{B} \in \mathcal{H}_{B}$,

$$
|\psi\rangle_{A B}=|\varphi\rangle_{A} \otimes|\chi\rangle_{B}
$$

we call it a separable state; otherwise, we call it an entangled state [86. Quantum entanglement is a uniquely quantum mechanical phenomenon which plays a key role in many interesting applications of quantum communication and quantum computation, and a detailed discussion can be found in the references [80] and [86].

\subsection{Quantum measurements}

\subsubsection{Projective measurement}

To better control a quantum system, it is often desirable to extract information from the controlled quantum system by means of measurements. Measurement theory in quantum mechanics is essentially different from that in classical mechanics since a measurement on a quantum system unavoidably affects the measured system (a detailed discussion of this issue can be found in [29]). In quantum mechanics, an observed physical quantity (called an observable) is expressed as a Hermitian operator on the Hilbert space $\mathcal{H}$ and one cannot simultaneously and precisely measure two noncommutative observables according to the Heisenberg uncertainty principle. A widely used measurement model is projective measurement (or von Neumann measurement). For an observable $M$, one can select a complete set of orthogonal projectors 
$\left\{P_{m}: \sum_{m} P_{m}=I, P_{m}=P_{m}^{\dagger}, P_{\tilde{m}} P_{m}=\delta_{\tilde{m}, m} P_{m}\right\}$ to describe a projective measurement, where $P_{m}$ is the projector onto the eigenspace of $M$ with eigenvalue $m, M=\sum_{m} m P_{m}$ and $\delta_{\tilde{m}, m}$ is the Kronecker delta [80]. For a quantum system in the state $|\psi\rangle$, the measurement outcome will correspond to one of the eigenvalues $m$ of the observable $M$. The probability of occurrence of outcome $m$ is $p(m)=\left\langle\psi\left|P_{m}\right| \psi\right\rangle$. Once the outcome $m$ has occurred, the state of the measured system changes to $\frac{P_{m}|\psi\rangle}{\sqrt{p(m)}}$ (this process is known as the collapse postulate). For a mixed state $\rho$, the probability of outcome $m$ is $p(m)=\operatorname{tr}\left\{P_{m} \rho\right\}$ and the state is changed into $\frac{P_{m} \rho P_{m}}{p(m)}$ if we obtain the outcome $m$.

\subsubsection{Continuous measurement}

Projective measurements are treated as instantaneous and this model is reasonable when the strength of the measurement (i.e., the coupling strength between the measurement apparatus and the measured system) is large enough and the measurement time-scale is much shorter than all other related time-scales for a given task [87]. However, this framework may not be sufficient to describe some important situations such as continuously monitoring some aspects of a quantum system [88, [89. In quantum feedback control, it is important to continuously extract feedback information to adjust the system evolution. Hence, there is an essential requirement to develop a continuous measurement theory for quantum feedback control. Fortunately, recent results have shown that continuous measurements are experimentally realizable for practical quantum systems such as solid-state qubits [90. In continuous measurements, one can continuously monitor an observable of a quantum system and the evolution of the system described in terms of the measurement record can be obtained from a stochastic master equation [89]. Continuous measurements can be derived from projective measurements under appropriate assumptions. For example, to implement a continuous measurement on an atom, we let the atom interact with an auxiliary quantum system (e.g., an electromagnetic field), and then make projective measurements on the auxiliary system (e.g., using a photodetector). In a short time interval, we extract only partial information about the atom. Hence, we also refer to continuous measurement as weak measurement or continuous weak measurement. For a detailed introduction to continuous measurement the reader can refer to [89]. Also, the stochastic master equation which describes a quantum system under continuous measurement will be presented in the following subsection.

\subsection{Quantum control models}

This subsection will introduce four types of models used in quantum control: bilinear models (BLM), Markovian master equations (MME), stochastic master equation models (SME) and linear quantum stochastic differential equations (LQSDE). 


\subsubsection{Bilinear models}

The state $|\psi(t)\rangle$ of a closed quantum system evolves according to the Schrödinger equation [84]

$$
i \hbar \frac{\partial}{\partial t}|\psi(t)\rangle=H_{0}|\psi(t)\rangle, \quad|\psi(t=0)\rangle=\left|\psi_{0}\right\rangle
$$

where $H_{0}$ is the free Hamiltonian of the system (i.e., a Hermitian operator on $\mathcal{H}$ ), $\hbar$ is the reduced Planck's constant (we assume that $\hbar=1$ in this paper), and the initial state has unit norm $\left\|\psi_{0}\right\|^{2} \equiv\left\langle\psi_{0} \mid \psi_{0}\right\rangle=1$. For simplicity, we consider finite dimensional quantum systems, which are appropriate approximations in many practical situations. For an $N$-dimensional quantum system, $\mathcal{H}$ is an $N$-dimensional complex Hilbert space, and the eigenstates $\left\{\left|\phi_{i}\right\rangle\right\}_{i=1}^{N}$ (denoting $\widetilde{D}=\left\{\left|\phi_{i}\right\rangle\right\}_{i=1}^{N}$ ) of $H_{0}$ form an orthogonal basis for $\mathcal{H}$. In many situations, the control of the system may be realized by a set of control functions $u_{k}(t) \in \mathbb{R}$ coupled to the system via time-independent Hermitian interaction Hamiltonians $H_{k}(k=1,2, \ldots)$. The total Hamiltonian $H(t)=H_{0}+\sum_{k} u_{k}(t) H_{k}$ then determines the controlled evolution

$$
i \frac{\partial}{\partial t}|\psi(t)\rangle=\left[H_{0}+\sum_{k} u_{k}(t) H_{k}\right]|\psi(t)\rangle .
$$

The goal in a typical quantum control problem defined on the system (5) is to find a final time $T>0$ and a set of admissible controls $u_{k}(t) \in \mathbb{R}$ which drives the system from the initial state $\left|\psi_{0}\right\rangle$ into a predefined target state $\left|\psi_{f}\right\rangle$. The total Hamiltonian $H(t)=H_{0}+\sum_{k} u_{k}(t) H_{k}$ defines a unitary transformation (propagator) $U(t)$ which can accomplish the transition from the pure state $\left|\psi_{0}\right\rangle$ to the pure state $|\psi(t)\rangle$; i.e.,

$$
|\psi(t)\rangle=U(t)\left|\psi_{0}\right\rangle
$$

Substituting (6) into (5), we can easily obtain

$$
i \dot{U}(t)=\left[H_{0}+\sum_{k} u_{k}(t) H_{k}\right] U(t), \quad U(0)=I .
$$

According to the quantum state superposition principle [83], the evolving state $|\psi(t)\rangle$ can be expanded in terms of the eigenstates in the set $\widetilde{D}$ :

$$
|\psi(t)\rangle=\sum_{j=1}^{N} c_{j}(t)\left|\phi_{j}\right\rangle .
$$

Substituting (8) into (15), we let $C(t)=\left\{c_{1}(t), c_{2}(t), \ldots, c_{N}(t)\right\}$ and obtain

$$
i \dot{C}(t)=\left[H_{0}+\sum_{k} u_{k}(t) H_{k}\right] C(t), \quad C_{0}=\left(c_{0 j}\right)_{j=1}^{N}, \quad c_{0 j}=\left\langle\phi_{j} \mid \psi_{0}\right\rangle .
$$

Equations (5), (7) or (9) are all referred to as finite dimensional bilinear models (BLM) of quantum control systems. If Equations (5), (77) and (9) are all controllable 
(see Section 3.1), conversion between them is easily carried out. For the BLM (77), we find a set of admissible controls to generate a desired unitary transformation $U(t)$ and then calculate the desired state trajectory using ([6). For the BLM (9), we find a set of admissible controls to achieve desired coefficient trajectories $C(t)$ and then obtain the desired state trajectory using (8) .

Bilinear models are widely used to describe closed quantum control systems such as molecular systems in physical chemistry and spin systems in NMR. For example, consider a spin $1 / 2$ system in a constant magnetic field along $z$-axis and controlled by magnetic fields along $x$-axis and $y$-axis [91]. We denote the Pauli matrices $\sigma=$ $\left(\sigma_{x}, \sigma_{y}, \sigma_{z}\right)$ as follows:

$$
\sigma_{x}=\left(\begin{array}{cc}
0 & 1 \\
1 & 0
\end{array}\right), \quad \sigma_{y}=\left(\begin{array}{cc}
0 & -i \\
i & 0
\end{array}\right), \quad \sigma_{z}=\left(\begin{array}{cc}
1 & 0 \\
0 & -1
\end{array}\right)
$$

The quantum control system under consideration can be described as follows:

$$
i \dot{U}(t)=\left[I_{z}+u_{x}(t) I_{x}+u_{y}(t) I_{y}\right] U(t), \quad U(0)=I
$$

where the controls $u_{x}(t), u_{y}(t) \in \mathbb{R}, I_{x}=\frac{1}{2} \sigma_{x}, I_{y}=\frac{1}{2} \sigma_{y}$ and $I_{z}=\frac{1}{2} \sigma_{z}$.

\subsubsection{Markovian master equations}

If we use the density matrix $\rho(t)$ to describe the state of a closed quantum system, the evolution equation for $\rho(t)$ can be described by the quantum Liouville equation

$$
i \dot{\rho}(t)=[H(t), \rho(t)],
$$

where $[X, \rho]=X \rho-\rho X$ is the commutation operator. In many practical applications, the quantum systems being considered are open quantum systems. In fact, this is the case for most quantum control systems since such systems unavoidably interact with their external environments (including control inputs and measurement devices). For an open quantum system, its evolution cannot generally be described in terms of a unitary transformation. In many situations, a quantum master equation for $\rho(t)$ is a suitable way to describe the dynamics of an open quantum system. One of the simplest cases is when a Markovian approximation can be applied where a short environmental correlation time is supposed and memory effects may be neglected [29]. For an $N$ dimensional open quantum system with Markovian dynamics, its state $\rho(t)$ can be described by the following Markovian master equation (MME) (for details, see, e.g., [29], 92], 93]):

$$
\dot{\rho}(t)=-i[H(t), \rho(t)]+\frac{1}{2} \sum_{j, k=0}^{N^{2}-1} \alpha_{j k}\left\{\left[F_{j} \rho(t), F_{k}^{\dagger}\right]+\left[F_{j}, \rho(t) F_{k}^{\dagger}\right]\right\} .
$$

Here $\left\{F_{j}\right\}_{j=0}^{N^{2}-1}$ is a basis for the space of linear bounded operators on $\mathcal{H}$ with $F_{0}=I$, the coefficient matrix $A=\left(\alpha_{j k}\right)$ is positive semidefinite and physically specifies the relevant relaxation rates. Markovian master equations have been widely used as models in Markovian quantum feedback (see Section 4.2). 


\subsubsection{Stochastic master equations}

In feedback control, we generally need to continuously monitor a quantum system to obtain feedback information. The evolution of a quantum system under continuous measurements of an observable $X$ can be described by the following master equation [89]:

$$
d \rho=-i[H, \rho] d t-\kappa[X,[X, \rho]] d t+\sqrt{2 \kappa}(X \rho+\rho X-2\langle X\rangle \rho) d W
$$

where $\kappa$ is a parameter related to the measurement strength, $\langle X\rangle=\operatorname{tr}(X \rho), d W$ is a Wiener increment with zero mean and variance equal to $d t$ and satisfies the following relationship to the measurement output $y$

$$
d W=d y-2 \sqrt{\kappa} \operatorname{tr}(X \rho) d t .
$$

Equation (14) is usually called a stochastic master equation (SME). Such a stochastic master equation can be obtained as a filtering equation from quantum filtering theory (for details, see [16], 62]). It is worth noting that the state $\rho$ in the SME (14) is a conditional state since it uses information from the continuous measurements. It should be pointed out that (14) is only a typical form of SME and there exist many different types of SMEs which depend on different measurement processes 94].

Stochastic master equations have been derived for some quantum optical systems under continuous measurements. For example, we consider an atomic ensemble interacting with an electromagnetic field considered in [16]. Consider the atomic Hamiltonian $H(t)=\Delta F_{z}+u(t) F_{y}$, where $\Delta$ is the atomic detuning and $u(t)$ is the strength of a magnetic field in the $y$-direction, and $F_{z}$ and $F_{y}$ are the collective dipole moments of the ensemble (for details, see [16]). If we neglect spontaneous emission, the corresponding $\mathrm{SME}$ is 16

$$
d \rho=-i\left[u(t) F_{y}+s F_{z}, \rho\right] d t-\kappa\left[F_{z},\left[F_{z}, \rho\right]\right] d t+\sqrt{2 \kappa}\left(F_{z} \rho+\rho F_{z}-2\left\langle F_{z}\right\rangle \rho\right) d W
$$

where $s$ is related to experimental parameters such as $\Delta$, and $d W$ satisfies

$$
d W=d y-2 \sqrt{\kappa} \operatorname{tr}\left(F_{z} \rho\right) d t
$$

\subsubsection{Linear quantum stochastic differential equations}

In the BLM (5), MME (13) and SME (14), we use the Schrödinger picture of quantum mechanics where equations describing the time dependence of quantum states are given. In some cases, it is more convenient to adopt the Heisenberg picture where the time-dependent operators on $\mathcal{H}$ are used in describing the quantum dynamics. An interesting case is a class of noncommutative linear stochastic systems which includes many examples of interest in quantum technology, especially in linear quantum optics [57. This class of systems can be described by the following linear quantum stochastic differential equation (LQSDE) [57]:

$$
\begin{aligned}
& d x(t)=A x(t) d t+B d w(t) ; x(0)=x_{0} \\
& d y(t)=C x(t) d t+D d w(t)
\end{aligned}
$$


where $A, B, C$ and $D$ are, respectively, $\mathbb{R}^{n \times n}, \mathbb{R}^{n \times n_{w}}, \mathbb{R}^{n_{y} \times n}$ and $\mathbb{R}^{n_{y} \times n_{w}}$ matrices $\left(n, n_{w}, n_{y}\right.$ are positive integers), and $x(t)=\left[x_{1}(t) \ldots x_{n}(t)\right]^{T}$ is a vector of self-adjoint possibly noncommutative system variables.

The initial system variables $x(0)=x_{0}$ consist of operators (on an appropriate Hilbert space) satisfying the commutation relations

$$
\left[x_{j}(0), x_{k}(0)\right]=2 i \Theta_{j k}, j, k=1, \ldots, n,
$$

where $\Theta_{j k}$ are the components of a real antisymmetric matrix $\Theta$. For simplicity, we may take $\Theta$ to have one of the following forms: (i) Canonical if $\Theta=\operatorname{diag}(J, \ldots, J)$, or (ii) degenerate canonical if $\Theta=\operatorname{diag}\left(0_{n^{\prime} \times n^{\prime}}, J, \ldots, J\right)$, where $0<n^{\prime} \leq n, n^{\prime}$ is the number of classical variables, and $J=\left[\begin{array}{cc}0 & 1 \\ -1 & 0\end{array}\right]$. The vector quantity $w$ describes the input signals and is assumed to admit the decomposition

$$
d w(t)=\beta_{w}(t) d t+d \tilde{w}(t)
$$

where $\tilde{w}(t)$ is the noise part of $w(t)$ and $\beta_{w}(t)$ is a self-adjoint, adapted process (see [95], [96]). The noise $\tilde{w}(t)$ is a vector of self-adjoint quantum noises with Itô table

$$
d \tilde{w}(t) d \tilde{w}^{T}(t)=F_{\tilde{w}} d t
$$

where $F_{\tilde{w}}$ is a positive semidefinite Hermitian matrix (see [95], 96] for details). Equation (18) can describe quantum systems such as linear quantum optical systems. However, it does not necessarily represent the dynamics of a meaningful physical system. We may need to add some additional constraints to ensure that a system described by equations of the form (18) is physically realizable (for details, see Section 4 or [57]).

\section{Controllability and open-loop control of quantum sys- tems}

\subsection{Controllability}

The controllability of quantum systems is a fundamental theoretical notion in quantum control as well as having practical importance because of its close connection with the universality of quantum computation [19] and the possibility of attaining atomic or molecular scale transformations [20, [21]. Different notions of controllability for quantum systems have been proposed and some notions have been studied in depth [25]. In this section, we will briefly present some aspects of controllability for quantum systems. A common research focus is on BLM of quantum systems for which the controllability criteria may be expressed in terms of the structure and rank of the corresponding Lie groups and Lie algebras [25]. We denote the $N$-dimensional complex unit sphere by $S_{\mathcal{C}}^{N}$ and the Lie algebra generated by the operators $\left\{-i H_{0},-i H_{1}, \ldots,-i H_{K}\right\}$ as $\mathcal{L}_{0}$. Also, we let $U(N)(S U(N))$ represent the $N$-dimensional unitary group (special unitary group) and $u(N)(s u(N))$ be the corresponding Lie algebra. Furthermore, we let $\mathcal{R}(|\psi\rangle)$ denote the reachable set of all states that are reachable from $|\psi\rangle$. Now, we give several definitions of controllability and their corresponding testing criteria. 
Definition 1 (Pure State Controllability) 24] The quantum system (5) is pure state controllable if for every pair of initial and final states, $\left|\psi_{0}\right\rangle$ and $\left|\psi_{f}\right\rangle$ in $S_{\mathcal{C}}^{N-1}$ there exist control functions $\left\{u_{k}(t)\right\}$ and a time $t>0$ such that the corresponding solution of (5) at time $t$, with initial condition $\left|\psi_{0}\right\rangle$, is $|\psi(t)\rangle=\left|\psi_{f}\right\rangle$.

Definition 2 (Operator Controllability) 24] The quantum system (7) is operator controllable if there exist control functions $\left\{u_{k}(t)\right\}$ which drive the unitary operator $U$ in (7) from $I$ to $U_{f}$, for any $U_{f} \in U(N)$ (or $\left.S U(N)\right)$.

Definition 3 (Eigenstate Controllability) [23] Suppose $\left|\phi_{1}\right\rangle, \ldots,\left|\phi_{N}\right\rangle$ are the $N$ eigenstates of the free Hamiltonian $H_{0}$. The quantum system (5) is eigenstate controllable if $\bigcup_{i=1}^{N} \mathcal{R}\left(\left|\phi_{i}\right\rangle\right)=S_{\mathcal{C}}^{N-1}$.

Theorem 4 [25] The quantum system (5) is pure state controllable if and only if the corresponding dynamical Lie algebra $\mathcal{L}_{0}$ satisfies one of the following conditions: (1) $\mathcal{L}_{0}=s u(N)$; (2) $\mathcal{L}_{0}$ is conjugate to $s p\left(\frac{N}{2}\right)$, where $s p\left(\frac{N}{2}\right)$ is the $\frac{N}{2}$-dimensional symplectic group; (3) $\mathcal{L}_{0}=u(N)$; (4) $\mathcal{L}_{0}=\operatorname{span}\left\{i \boldsymbol{1}_{N \times N}\right\} \oplus \tilde{\mathcal{L}}$, where $\tilde{\mathcal{L}}$ is a Lie algebra conjugate to $\operatorname{sp}\left(\frac{N}{2}\right)$.

Theorem 5 24 The quantum system (7) is operator controllable if and only if $\mathcal{L}_{0}=u(N) \quad\left(\right.$ or $\left.\mathcal{L}_{0}=s u(N)\right)$.

From the above definitions and theorems, we can see that the notion of operator controllability is strongest of these quantum controllability notions and eigenstate controllability is weakest. If a quantum system is operator controllable, it is easy to show that it is also pure state controllable using (6) or by a comparison of Theorems 4 and 5. If a quantum system is pure state controllable, it follows from the definitions that it must also be eigenstate controllable. Note that pure state controllability is also called wavefunction controllability in some references (e.g., [27, [28], [43]). Operator controllability, in the unitary case, is also called complete controllability [22, [97]. Some algorithms have been developed for testing the controllability of specific quantum systems [20], 22], 25], 98].

The Lie algebra method allows for a straightforward mathematical treatment of closed quantum systems. However, the relevant criteria may be computationally difficult when the dimension of the controlled system is large. Hence, another method based on graph theory has been developed for pure state controllability for which the controllability criterion becomes easy to verify. For simplicity, we consider the quantum system (9) with only a single control input $u(t)$; i.e.,

$$
i \dot{C}(t)=A C(t)+u(t) B C(t) \quad C_{0}=\left(c_{0 j}\right)_{j=1}^{N}
$$

where the matrices $A$ and $B$ correspond to the free Hamiltonian and interaction Hamiltonian, respectively. Now, we associate the system with a non-oriented connectivity graph $G=(V, E)$, where the set of vertices $V$ corresponds to the set of the eigenstates 
$\left|\phi_{i}\right\rangle$ and the set of edges $E$ corresponds to the set of all pairs of eigenstates directly coupled by the matrix $B=\left(B_{i j}\right)$,

$$
\begin{aligned}
G(V, E): \quad V & =\left\{\left|\phi_{1}\right\rangle, \ldots,\left|\phi_{N}\right\rangle\right\}, \\
E & =\left\{\left(\left|\phi_{i}\right\rangle,\left|\phi_{j}\right\rangle\right) ; i<j, B_{i j} \neq 0\right\} .
\end{aligned}
$$

Let $G_{k}=\left(V^{(k)}, E^{(k)}\right), k=1, \ldots, K$ be connected components of this graph. We denote by $\lambda_{i}(i=1, \ldots, N)$ the eigenvalues of the matrix $A$ and let $\nu_{i j}=\lambda_{i}-\lambda_{j}$ $(i, j=1, \ldots, N)$. The following theorem provides a sufficient condition for pure state controllability in terms of the connectivity graph [27, [28]:

Theorem 6 The system (22) is pure state controllable if the following conditions hold:

(I) The graph $G$ is connected; i.e. $K=1$.

(II) The graph $G$ does not have "degenerate transitions". That is for all $(i, j) \neq(a, b)$, $i \neq j, a \neq b$ such that $B_{i j} \neq 0, B_{a b} \neq 0$, then $\nu_{i j} \neq \nu_{a b}$.

(III) For each $i, j, a, b=1, \ldots, N$ such that $\nu_{i j} \neq 0$ the number $\left(\nu_{a b} / \nu_{i j}\right)$ is rational.

The proof of Theorem [6] can be found in [28]. The three conditions in Theorem [6 provide a sufficient but not a necessary condition for pure state controllability. In some circumstances, the assumptions (II) and (III) can be slightly relaxed [27]. Moreover, it is clear that if we add one more eigenstate into the set $\widetilde{D}$, the pure state controllability criterion is easy to check for the resultant new system [27.

All of the above results consider finite dimensional quantum systems, which are a reasonable approximation to the quantum systems arising in many applications. Many practical quantum systems, especially those with continuous spectra, are essentially infinite dimensional; i.e., $|\psi(t)\rangle$ in (5) is a unit vector in an infinite dimensional Hilbert space $\mathcal{H}$. The controllability of such infinite dimensional quantum systems has been studied in [3]. For an infinite dimensional system (5), the existence of a dense analytic domain (in the sense of Nelson), together with some conditions on the corresponding Lie algebra, can guarantee its analytic controllability. The controllability of infinite dimensional quantum control systems has also been extensively studied in [21, 99].

Another interesting focus is on the controllability of open quantum systems. It has been proven that a finite dimensional open quantum system with Markovian dynamics (i.e., MME (13)) is not controllable when using only coherent control 30. However, finite dimensional open quantum systems with Kraus-map dynamics are complete kinematic state controllable [26] and a specific Kraus map can be constructed for transformation from an arbitrary initial state to a predefined target state if some incoherent resources are available as control tools [100], [101]. A control approach involving generating Kraus type dynamics can be potentially robust to variations of the initial system state [26].

\subsection{Optimal control}

Most results on the controllability of quantum systems do not provide constructive methods to design a control law for driving a quantum system from an initial state to a 
predetermined target state. Hence, it is desirable to develop useful methods to design such a control law. A straightforward strategy for constructing a suitable control law is based on a Lie group decomposition, where the desired system evolution operator $U_{f}$ is decomposed as the product of basic unitary operators which are easy to generate using simple controls [25], [102], [103]. This method is constructive and exact. However, it may be difficult to complete the decomposition for most practical systems. For many practical quantum control problems, optimal control theory is a powerful tool in achieving quantum control objectives [33, [104].

In the optimal control approach, the quantum control problem can be formulated as a problem of seeking a set of admissible controls satisfying the system dynamic equations and simultaneously minimizing a cost functional. The cost functional may be different according to the practical requirements of the quantum control problem, such as minimizing the control time [35, [105, the control energy [91, 106], the error between the final state and target state, or a combination of these requirements. Many useful tools in traditional optimal control, such as the variational method, the Pontryagin minimum principle and convergent iterative algorithms [107, can be adapted to quantum systems and applied to search for optimal controls. Optimal control techniques have been widely applied to control quantum phenomena in physical chemistry (for details, see, e.g., 9], [11, [12], [108], [109]) and NMR experiments [39], [40], 81].

Here we present a simple example to demonstrate optimal quantum control design. We consider the model (11) and assume $u_{y}(t)=0$. We can produce an $x$ rotation of the spin by using radio-frequency $(\mathrm{RF})$ pulses to control the system. The quantum control system becomes

$$
i \dot{U}(t)=\left[I_{z}+u_{x}(t) I_{x}\right] U(t), \quad U(0)=I
$$

From Theorem 5, it is easy to check that the system (24) is operator controllable (completely controllable). It is possible to design a control law which is optimal in some sense to accomplish a specified quantum control task. In some applications, it is desirable to accomplish the control task as soon as possible in order to minimize the relaxation effects [35. For example, it is desirable to perform a quantum computation task with a minimum time using a collection of control resources [110]. Hence, the time-optimal control of quantum systems is an interesting practical problem [35], 105]. If the control is not bounded, we have the following theorem [35].

Theorem 7 Consider the model (24). Given any $U_{f} \in S U(2)$, there exists a unique $\beta \in[0,2 \pi]$ such that $U_{f}=\exp \left(-i \alpha I_{x}\right) \exp \left(-i \beta I_{z}\right) \exp \left(-i \gamma I_{x}\right)$, where $\alpha, \gamma \in \mathbb{R}$, and the minimum time required in producing $U_{f}$ is $\beta$.

If we ignore the global phase, we can denote the initial state $\left|\psi_{0}\right\rangle$ and the target state $\left|\psi_{f}\right\rangle$ as

$$
\begin{aligned}
& \left|\psi_{0}\right\rangle=\cos \frac{\theta_{0}}{2}|0\rangle+e^{i \varphi_{0}} \sin \frac{\theta_{0}}{2}|1\rangle, \\
& \left|\psi_{f}\right\rangle=\cos \frac{\theta_{f}}{2}|0\rangle+e^{i \varphi_{f}} \sin \frac{\theta_{f}}{2}|1\rangle
\end{aligned}
$$

where $\theta_{0}, \theta_{f} \in[0, \pi], \varphi_{0}, \varphi_{f} \in[0,2 \pi]$ and $\left|\psi_{f}\right\rangle=U_{f}\left|\psi_{0}\right\rangle$. We can give an analytical expression for the minimal time required to accomplish this control task using geometric 
manipulations on the Bloch sphere [52]. This minimum time $\beta$ can be calculated according to the following equation:

$$
\beta=\left|\arccos \left(\sin \theta_{f} \cos \varphi_{f}\right)-\arccos \left(\sin \theta_{0} \cos \varphi_{0}\right)\right| .
$$

A formal proof of this result can be found in [53].

Under the assumption of bounded control (i.e., $|u(t)| \leq V \in \mathbb{R}^{+}$), an interesting case is bang-bang control where the control $u(t)$ switches between two values $\pm V$. The solutions to many optimal problems take the form of bang-bang control [37. The bang-bang control strategy has been used to suppress decoherence [111] (the evolution of pure states to mixed states; e.g., see [86]) and to manipulate a class of coherent states [112]. To reduce the error introduced by the switches, the number of switches can be used as a performance index for optimal control design. Several algorithms have been developed to determine the minimum number of switches required. In one approach, the number of switches is determined by finding the minimum number of factors required in the factorization of $U_{f}$ [113]. Another approach involves geometric analysis on the corresponding complex unit sphere (for details, see [53]).

From the above example, we can see that, for the quantum system (24), we may wish to optimize different cost functionals depending on the application. We may also use different methods to solve the same optimal quantum control problem. Optimal control is often combined with other strategies, such as closed-loop learning control and quantum feedback control, when manipulating quantum entanglement [69], identifying Hamiltonian parameters [76], controlling chemical reactions [9], [12], and tracking quantum states [114].

\subsection{Lyapunov-based control design}

Lyapunov-based control methods are powerful tools for feedback controller design in classical control theory. In quantum control, the acquisition of feedback information through measurements usually destroys the state being measured, which makes it difficult to directly apply Lyapunov approaches to quantum feedback controller design. However, we may first complete the feedback control design by simulation on a computer, which will give a sequence of controls. Then we can apply the control sequence to the quantum system to be controlled in an open-loop form [41], [42], [115]-121]. This is a "feedback design and open-loop control" strategy. This strategy is especially useful for some difficult quantum control tasks [120]. The most important aspects in Lyapunov-based control design include the construction of the Lyapunov function, the determination of the control law and the analysis of asymptotic convergence.

To design an open-loop control law for quantum systems, several types of Lyapunov functions have been considered. For example, if the target state is $\left|\psi_{f}\right\rangle$, we may select one of the following Lyapunov functions: $V_{1}(t)=\frac{1}{2}\left(1-\left|\left\langle\psi_{f} \mid \psi(t)\right\rangle\right|^{2}\right)$ [116], $V_{2}(t)=$ $\left\langle\psi(t)-\psi_{f} \mid \psi(t)-\psi_{f}\right\rangle$ [41], or $V_{3}(t)=\langle\psi(t)|P| \psi(t)\rangle$, where $P$ is a positive semidefinite Hermitian operator [117]. It is clear that $V_{j}(t) \geq 0(j=1,2,3)$. We can now select the control function to guarantee that the first-order time derivative of the Lyapunov 
function is negative semidefinite. That is, we may determine the control law using the condition $\dot{V}(t) \leq 0$ on the Lyapunov function $V(t)$.

For the case that the target state is an eigenstate of the free Hamiltonian $H_{0}$, several algorithms have been developed to find corresponding control laws and their convergence has been analyzed [42], [116], [117]. For the case that target states are not eigenstates of $H_{0}$, the control problem can be formulated in terms of reference trajectory tracking [41], [118], 122]. Lyapunov-based techniques have also been formulated using density operators for the control of a spin ensemble [120, [121. LaSalle's invariance principle is also useful to characterize the asymptotic behavior of the system dynamics [25].

\subsection{Variable structure control}

For some quantum systems which are not controllable, we can introduce additional resources or special strategies to change the controllability of the quantum system or enhance our capability of controlling the quantum system. In this subsection, we introduce a variable structure control strategy to enhance our capability of controlling a class of quantum systems. In variable structure control, one can change the controller structure according to specified switching logic in order to obtain improved properties 43.

Consider an example corresponding to two special cases of (91):

$$
\begin{aligned}
i \dot{C}(t) & =A C(t)+u_{1}(t) B_{1} C(t)=\left(A+B_{1}^{u}\right) C(t) ; \\
i \dot{C}(t) & =A C(t)+u_{2}(t) B_{2} C(t)=\left(A+B_{2}^{u}\right) C(t)
\end{aligned}
$$

where $A=\operatorname{diag}\{1.0,1.2,1.3,2.0,2.15\}$, and

$$
B_{1}^{u}=\left(\begin{array}{ccccc}
0 & 0 & 0 & u_{1}(t) & u_{1}(t) \\
0 & 0 & 0 & 0 & 0 \\
0 & 0 & 0 & 0 & 0 \\
u_{1}(t) & 0 & 0 & 0 & u_{1}(t) \\
u_{1}(t) & 0 & 0 & u_{1}(t) & 0
\end{array}\right) ; \quad B_{2}^{u}=\left(\begin{array}{ccccc}
0 & 0 & u_{2}(t) & 0 & 0 \\
0 & 0 & 0 & u_{2}(t) & u_{2}(t) \\
u_{2}(t) & 0 & 0 & 0 & 0 \\
0 & u_{2}(t) & 0 & 0 & u_{2}(t) \\
0 & u_{2}(t) & 0 & u_{2}(t) & 0
\end{array}\right)
$$

In [43, it has been shown that the two models (28) and (29) are not individually pure state controllable but are controllable under a variable structure control strategy. Furthermore, consider a system $\left(S_{m}\right)$ :

$$
i \dot{C}(t)=\left(A+B_{m}^{u}\right) C(t) \quad(m=1, \ldots, M)
$$

The corresponding connectivity graph of $B_{m}^{u}$ is denoted as $G_{m}=\left(V_{m}, E_{m}\right)$. It is clear that for arbitrary $m, V_{m}=V=\left\{\left|\phi_{1}\right\rangle, \ldots,\left|\phi_{N}\right\rangle\right\}$. Hence, we can write $G_{m}=\left(V, E_{m}\right)$ and define $G=(V, E)$ as the combined graph of $G_{m}=\left(V, E_{m}\right)(m=1, \ldots, M)$ where $E=E_{1} \cup E_{2} \cup \cdots \cup E_{M}$. We then give the following theorem [44].

Theorem 8 The system

$$
i \dot{C}(t)=\left(A+B^{u}\right) C(t) \quad C(0)=C_{0} \quad B^{u} \in\left\{B_{1}^{u}, \ldots, B_{M}^{u}\right\}
$$


is pure state controllable if the controller $B^{u}$ can be arbitrarily switched between controllers $B_{m}^{u}$ and $B_{n}^{u}(m \neq n, 1 \leq m, n \leq M)$, and the following assumptions hold:

(I) The combined graph $G$ for the system (31) is connected.

(II) The combined graph $G$ remains connected after elimination of edge pairs $\left(\left|\phi_{j}\right\rangle\right.$,

$\left.\left|\phi_{l}\right\rangle\right),\left(\left|\phi_{a}\right\rangle,\left|\phi_{b}\right\rangle\right)$ such that $\nu_{j l}=\nu_{a b}$ (degenerate transitions).

The proof of this theorem can be found in [44. The variable structure control strategy provides a useful method for open-loop control design for a class of quantum systems and is also applicable to qubit preparation for quantum information processing (for details, see [43], 44]). Here, for open-loop control of quantum systems, we only use the concept of variable structure control and consider control inputs which are allowed to switch between a number of different control structures. Moreover, note that sliding mode control is an important aspect in variable structure control of classical systems. Also, the sliding mode method can be used to deal with uncertainties in the feedback control of quantum systems and has potential applications to quantum state preparation and quantum error correction [123].

\subsection{Incoherent control}

The above discussions on open-loop quantum control focus on coherent control where we can manipulate the state of a quantum system by applying semiclassical potentials in a fashion that preserves quantum coherence [17]. The control inputs commonly occur as tunable parameters in the Hamiltonian of the system and can directly affect the coherent part of the dynamics. There exist some physical situations where it is not possible or very difficult to control the state of a quantum system directly with coherent resources (unitary transformations) [49]. For example, it has been proven that a finite dimensional open quantum system with Markovian dynamics is not controllable using only coherent control [30, [124]. A natural extension of these quantum control methods is to introduce incoherent resources (called "incoherent control") to enhance our capability of controlling quantum systems or to help in control design in order to accomplish specified tasks. The main paradigms for introducing incoherent resources into open-loop quantum control include incoherent control fields [12, the use of auxiliary systems (environments) and quantum measurements [45]-[55], [125]-127].

Quantum measurements are regarded as deleterious in accomplishing coherent control tasks since they usually destroy the coherent characteristics of the quantum system. However, recent results show that quantum measurements can improve the controllability of quantum systems in some situations. Indeed, sometimes a quantum system which is not unitarily controllable can be controlled by the joint action of projective measurement and unitary evolution [45, 48]. Let us consider the model (5), and let $G(\mathcal{A})$ denote the Lie group generated by the operators $\left\{-i H_{0},-i H_{1}, \ldots,-i H_{K}\right\}$. Also, let $O(N)$ be the $N$-dimensional orthogonal group. We then have the following theorem [45]:

Theorem 9 Given any goal state $\left|\psi_{f}\right\rangle$, there is a family of observables $M\left(\left|\psi_{f}\right\rangle\right)$ such that measurement of one of these observables on any $\left|\psi_{0}\right\rangle$ plus unitary evolution 
leads to $\left|\psi_{f}\right\rangle$ if $G(\mathcal{A})$ is equal to either $O(N)$ or $\operatorname{sp}\left(\frac{N}{2}\right)$.

The proof of this theorem can be found in [45]. If $G(\mathcal{A})$ is equal to either $U(N)$ or $s p\left(\frac{N}{2}\right)$, the corresponding system is pure state controllable and it might still be more efficient to use the measurement plus evolution strategy [45]. Several incoherent control schemes based on quantum measurements have been proposed. For example, a control scheme for mapping an unknown mixed quantum state onto a known pure state has been proposed with the help of sequential measurements of two noncommutative observables and without the use of unitary transformations [125]. A probabilistic quantum control strategy using indirect measurement is presented for the remote control of quantum systems [48]. Projective measurements on several identical initial states are used to help complete the control design of quantum systems with pure state controllable subspaces [51. Projective measurements are also combined with amplitude amplification for the state control of locally controllable quantum systems [55. In these incoherent control schemes, we emphasize the role of measurement as a control tool. Although we may use the measurement result, we present these methods as open-loop control strategies since no real closed-loop is constructed in the control process.

Quantum measurements can be used as an effective control tool in quantum control. In practical applications, the realization of a quantum measurement on a quantum system is usually accomplished by entangling the quantum system with an auxiliary system (a probe). Hence, one can also control a quantum system by controlling an auxiliary system (e.g., a probe) entangled with the quantum system. Under suitable conditions on the interaction of the system to be controlled, the auxiliary system, and the environment, the system dynamics is completely controllable by varying the initial state of the auxiliary system [49], [50] or manipulating the local Hamiltonian of the auxiliary system [128, which has potential applications to quantum networks [129]. Furthermore, if all kinds of coherent and incoherent resources are available, in principle, we can control a (closed or open) quantum system from an arbitrary initial state to a predefined target state [100, [101].

\section{Some results on closed-loop design methods}

As presented in Section 3, open-loop control has achieved many successes in the control of some simple quantum systems. However, it suffers some difficulties for more complex quantum control tasks such as suppressing decoherence and dealing with disturbances in quantum systems. A natural solution to this problem is to explore closed-loop control strategies. Two forms for closed-loop control have been proposed for quantum systems: closed-loop learning control and quantum feedback control. In closed-loop learning control, each cycle of the closed-loop is executed on a new sample. However, in quantum feedback control, the same sample is involved during the whole process of control [9]. In this section, we will present some aspects of quantum feedback control after a brief introduction to closed-loop learning control. 


\subsection{Closed-loop learning control}

Closed-loop learning control has achieved great successes in the control of laboratory chemical reactions [9], [58. The closed-loop learning control procedure generally involves three elements [9]: (i) a trial laser control input design, (ii) the laboratory generation of the control that is applied to the sample and subsequently observed for its impact, and (iii) a learning algorithm that considers the prior experiments and suggests the form of the next control input. The initial trial control input may be a well-designed laser pulse or a random control input. A feature of a good closed-loop learning control strategy is its insensitivity to the initial trials. An important task is to establish a good learning algorithm for ensuring that the closed-loop learning process converges to achieve a given control objective. Genetic algorithms and several rapid convergence algorithms have been employed for this task [58], [107]. The control objective is usually

formulated as an optimal control problem by converting the problem into a problem of optimizing a functional of the quantum states, control inputs, control time, etc. In the learning process, an optimal control problem is solved iteratively. First, one applies a trial input to a sample to be controlled and observes the result. Second, a learning algorithm suggests a better control input based on the prior experiments. Third, one applies a "better" control input to a new sample. This process continues in order to achieve the control objective. It is often easy to produce many identical-state samples in laboratory chemical reactions. If the control objective is well defined, there is a capability to apply specified control inputs to the samples. Then, if a sufficiently intelligent learning algorithm is applied to adjust the control inputs, this process will converge to optimize the required objective and an optimal control will be found [9].

\subsection{Quantum feedback control}

As we know, feedback is an effective strategy in classical control theory and the aim of feedback is to compensate for the effects of unpredictable disturbances on a system under control, or to make automatic control possible when the initial state of the system is unknown. In classical control, many results have shown that feedback control is superior to open-loop control. In feedback control, it is usually necessary to obtain the information about the state of system through measurement. However, measurements of a quantum system will unavoidably disturb the state of measured quantum system, which makes the situation more complex when applying feedback to quantum systems. In spite of this difficulty, important progress has been made and feedback has been used to improve the control performance for squeezed states [60], [130], quantum entanglement [131], [132, [133], and quantum state reduction [62]-65], [134-[138] in many areas such as quantum optics [15], 82], superconducting quantum systems [138], Bose-Einstein condensate [139] and nanomechanical systems [140]. Quantum feedback control has also been compared with open-loop control (e.g., see [141]). It has been proven for a specific quantum system that quantum feedback is superior to open-loop control in dealing with uncertainties in initial states and it has also been demonstrated via simulation that feedback control is still better than open-loop control for dealing 
with decoherence [141.

In quantum feedback control, the two main approaches to information acquisition are projective measurement and continuous weak measurement. The system to be controlled is a quantum system, however, the controller may be quantum, classical or a quantum/classical hybrid. Several paradigms of quantum feedback have been proposed, such as Markovian quantum feedback [15, [142, Bayesian quantum feedback [73, 143], 144, and coherent quantum feedback [17], [57]. In Markovian quantum feedback, any time delay is ignored and a memoryless controller is assumed. That is, the measurement record is immediately fed back onto the system to alter the system dynamics and may then be forgotten [145]. Hence, the equation describing the resulting evolution is a Markovian master equation. In Bayesian quantum feedback, the process is divided into two steps involving state estimation and feedback control. The best estimates of the dynamical variables are obtained continuously from the measurement record, and fed back to control the system dynamics [73. The dynamical equation describing the resulting evolution is non-Markovian. In coherent quantum feedback, the feedback controller itself is a quantum system, and it processes quantum information. This is greatly different from Markovian and Bayesian quantum feedback where the feedback information from measurement results is classical information and the feedback controller is a classical controller. In the following, we will present several important aspects of quantum feedback control including quantum filtering, feedback stabilization, optimal feedback control and robust control.

\subsubsection{Identification and filtering/estimation}

To design an effective quantum feedback control system, it is necessary to obtain knowledge of a system model and system states. This can be regarded as a quantum system identification or state estimation problem. The simplest example is to estimate the system state for feedback through projective measurements on a quantum ensemble. This is a simple problem in classical control which can usually be accomplished through direct measurements. However it becomes much more complex due to the quantum collapse postulate of measurement in quantum control. This problem of identifying quantum states is also called quantum state tomography in quantum information and some procedures have been developed to experimentally determine an unknown quantum state [80]. Some of these procedures can be used in quantum feedback control. Another example is the identification of parameters for a quantum operation (e.g., a quantum gate or the system Hamiltonian). This procedure is called as quantum process tomography and some techniques have been developed (e.g., see [76], [80]). To acquire information about a quantum operation, an attractive approach is quantum nondemolition measurements which do not disturb the value to be measured. However, it is not practical to design quantum nondemolition measurements for most problems (see [146] for details).

Furthermore, it is often useful to estimate an unknown dynamical parameter through continuously monitoring an open quantum system [147], [148]. Since continuous observations of open quantum systems are inherently noisy, quantum filtering theory is 
required for extracting information from a noisy signal [71. Quantum filtering theory has been developed for quantum optical systems and is used as an essential component in some quantum feedback control strategies [70], [72], [96], [149]. In the framework of noncommutative (or quantum) probability theory, a broad class of quantum stochastic differential equations can be obtained (for details, see, e.g., [71]). In fact, the SME model (14) is a quantum filtering equation which describes the evolution of a conditional state. Recently, several robustness properties of quantum filtering and estimation have also been investigated for linear and nonlinear quantum systems [75, [150], 151].

\subsubsection{Feedback stabilization/control}

Based on the use of feedback information from measurements or estimation, one can design a feedback controller to control or stabilize a quantum system. In Markovian quantum feedback, the feedback Hamiltonian is commonly selected as a linear function of the feedback signal. For example, the feedback Hamiltonian in Markovian feedback control via homodyne detection may be selected as $H_{f b}(t)=F(t) I(t)$, where $F(t)$ is a Hermitian operator and $I(t)$ is a current from the measurement [15, [145, [152]. Then the feedback Hamiltonian will enter the system Hamiltonian to alter the system evolution. Markovian quantum feedback has been widely applied in the stabilization of arbitrary pure states of two-level systems [152], noiseless subspace generation [153, continuous quantum error correction [154, etc. In Bayesian quantum feedback, the feedback Hamiltonian is a general function of the measurement record [73], [155], which is used to control the system dynamics. Bayesian feedback is usually superior to Markovian feedback since it uses more information. However, it is more difficult to implement Bayesian feedback than Markovian feedback due to the existence of the estimation step [145]. Bayesian feedback has also been applied in the preparation of quantum states [155] and quantum error correction [66, 67]. If the feedback delay cannot be ignored, the feedback Hamiltonian must include a delay parameter [156] which in some cases has a qualitatively similar effect to that of unprecise measurement [157]. Both Markovian feedback and Bayesian feedback use information from measurement (or estimation) and the feedback controller is usually a classical controller. Another different feedback paradigm is coherent quantum feedback, where a quantum controller (i.e., the controller is another quantum system) directly affects the system dynamics to be controlled [17, [158, [159].

In quantum feedback control, the filtering SME is an important dynamical equation for the design of the feedback control system. For example, for a quantum system described by an SME, one may use stochastic Lyapunov techniques to design a feedback control law for asymptotically stabilizing the quantum system to an objective state. For the model (16), if $\mathcal{S}=\left\{\rho \in \mathbb{C}^{N \times N}: \rho=\rho^{\dagger}, \operatorname{tr} \rho=1, \rho \geq 0\right\}$, we have the following result [63]:

Theorem 10 Consider the system (16) evolving in the set $\mathcal{S}$. Let the final state

$\rho_{f}=v_{f} v_{f}^{\dagger}$, where $v_{f}$ is an eigenstate $\left|\phi_{m}\right\rangle(1 \leq m \leq N)$, and let $\gamma>0$. Consider the following control law: 
1. $u_{t}=-\operatorname{tr}\left(i\left[F_{y}, \rho_{t}\right] \rho_{f}\right)$ if $\operatorname{tr}\left(\rho_{t} \rho_{f}\right) \geq \gamma$.

2. $u_{t}=1$ if $\operatorname{tr}\left(\rho_{t} \rho_{f}\right) \leq \gamma / 2$.

3. If $\rho_{t} \in \mathcal{B}=\left\{\rho: \gamma / 2<\operatorname{tr}\left(\rho \rho_{f}\right)<\gamma\right\}$, then $u_{t}=-\operatorname{tr}\left(i\left[F_{y}, \rho_{t}\right] \rho_{f}\right)$ if $\rho_{t}$ last entered $\mathcal{B}$ through the boundary $\operatorname{tr}\left(\rho \rho_{f}\right)=\gamma$, and $u_{t}=1$ otherwise.

Then there exists a $\gamma>0$ such that $u_{t}$ globally stabilizes (16) around $\rho_{f}$ and $\mathbb{E} \rho_{t} \rightarrow \rho_{f}$ as $t \rightarrow \infty$.

\subsubsection{LQG control}

Under appropriate assumptions, some quantum optical systems can be approximately modeled by LQSDEs driven by quantum Wiener processes [56], [160]. This simplification provides an opportunity to develop quantum linear-quadratic-Gaussian (LQG) control to obtain an optimal feedback strategy [4], 69], [70], [73], [143], [160], [161, 162. In LQG control, the goal is to find an optimal feedback control law for a stochastic linear system by optimizing a quadratic cost functional. In some cases, results from classical LQG control can be applied to quantum systems [163]. An important result is the separation theorem which applies when the system is linear, the cost functional is quadratic in the system variables and the noises are Gaussian. In this situation, the values of optimal estimates are fed back. Then, when calculating the feedback required for the optimal control, we may assume that the dynamical variables are known exactly [73.

In the quantum LQG control problem, the optimal control is also a linear feedback control law. The controller may be a classical controller [162] or a quantum controller [158, [159]. For classical controllers, it is straightforward to apply results in classical LQG control to quantum LQG problems. In fact, some results on quantum LQG control with classical controllers have been developed in [69], [73], [143], [160], [162]. For controllers which are themselves quantum systems, we must add some additional constraints on coefficient matrices to ensure that the controller is physically realizable. For example, [57] has developed a notion of physical realizability based on the concept of an open quantum harmonic oscillator as the basic components of a physically realizable quantum system. Under several reasonable assumptions on a system of the form (18) (e.g., $n_{y}$ is even, $n_{\omega} \geq n_{y}, F_{\omega}=I+i \operatorname{diag}(J, \ldots, J)$, etc.; see [57] for details), we have the following results on physical realizability [57]:

Theorem 11 The system (18) is physically realizable if and only if:

$$
\begin{gathered}
i A \Theta+i \Theta A^{T}+B T_{\omega} B^{T}=0, \\
B\left[\begin{array}{c}
I_{n_{y} \times n_{y}} \\
0_{\left(n_{\omega}-n_{y}\right) \times n_{y}}
\end{array}\right]=\Theta C^{T} \operatorname{diag}_{N_{y}}(J) \\
D=\left[I_{n_{y} \times n_{y}} \quad 0_{n_{y} \times\left(n_{\omega}-n_{y}\right)}\right]
\end{gathered}
$$

where $N_{y}=\frac{n_{y}}{2}$ and $T_{\omega}=\frac{1}{2}\left(F_{\omega}-F_{\omega}^{T}\right)$. 
Hence, the problem of quantum LQG control with a quantum controller is converted into the following problem: Given a canonical commutation relation, find a feedback controller, whose coefficient matrices satisfy the conditions of physical realizability in Theorem 11, that minimizes a quadratic performance index. The additional constraints on the coefficients for controllers make the problem more complex. Indeed, new algorithms are required to be developed in order to solve this problem. In [159], a numerical procedure based on an alternating projection algorithm has been developed to solve this control problem. Furthermore, several results on synthesizing coherent quantum controllers have also been presented (e.g., see [164]).

\subsubsection{Robust control}

In practical applications, it is unavoidable that quantum systems are subject to all kinds of disturbances and uncertainties [165. Many instances of incomplete knowledge and unknown errors can also be treated as uncertainties. Hence, robustness has been identified as an important aspect for the practical application of quantum technology [79], [166], [167]. Recently, several robust control methods in classical control theory have been extended into quantum domain. For example, the small gain theorem has been applied to the stability analysis of quantum feedback networks [79]. A transfer function approach has been applied to the feedback and robust control for single input single output quantum systems [168, [169]. An $H^{\infty}$ controller synthesis problem has been formulated for a class of linear quantum stochastic systems [57]. A sliding mode control approach has been applied to robust control design for quantum systems where bounded uncertainties exist in the system Hamiltonian [123. Some open-loop and feedback approaches to quantum control can lead to a certain degree of robustness. Since feedback plays a key role in robust control, in this survey we present robust control within the framework of quantum feedback control. In the following, we will only present several specific results on $H^{\infty}$ control for quantum systems, which is based on the reference [57].

Consider the quantum system to be controlled to be described by the following LQSDE defined in an analogous way to (18):

$$
\begin{aligned}
& d x(t)=A x(t) d t+\left[\begin{array}{lll}
B_{0} & B_{1} & B_{2}
\end{array}\right] \times\left[\begin{array}{lll}
d v(t)^{T} & d w(t)^{T} & d u(t)^{T}
\end{array}\right]^{T} ; x(0)=x_{0} \\
& d z(t)=C_{1} x(t) d t+D_{12} d u(t) \\
& d y(t)=C_{2} x(t) d t+\left[\begin{array}{lll}
D_{20} & D_{21} & 0_{n_{y} \times n_{u}}
\end{array}\right] \times\left[\begin{array}{lll}
d v(t)^{T} & d w(t)^{T} & d u(t)^{T}
\end{array}\right]^{T} .
\end{aligned}
$$

where the input $w(t)$ represents a disturbance signal of the form (20). Also, $d v(t)$ represents any additional quantum noise in the system. The control input $u(t)$ can be written as

$$
d u(t)=\beta_{u}(t) d t+d \tilde{u}(t)
$$

where $\beta_{u}(t)$ is the adapted, self-adjoint part of $u(t)$ and $\tilde{u}(t)$ is the noise part of $u(t)$. The vectors $v(t), \tilde{w}(t)$, and $\tilde{u}(t)$ are quantum noises with positive semidefinite Hermitian Itô matrices $F_{v}, F_{\tilde{w}}$ and $F_{\tilde{u}}$. 
The controller is assumed to be of the following form

$$
\begin{aligned}
& d x(t)=A_{K} \xi(t) d t+\left[\begin{array}{ll}
B_{K 1} & B_{K}
\end{array}\right] \times\left[\begin{array}{ll}
d v_{K}(t)^{T} & d y(t)^{T}
\end{array}\right]^{T} ; \xi(0)=\xi_{0} \\
& d u(t)=C_{K} \xi(t) d t+\left[\begin{array}{ll}
B_{K 0} & 0_{n_{u} \times n_{y}}
\end{array}\right] \times\left[\begin{array}{ll}
d v_{K}(t)^{T} & d y(t)^{T}
\end{array}\right]^{T}
\end{aligned}
$$

where $\xi(t)=\left[\xi_{1}(t) \ldots \xi_{n}(t)\right]^{T}$ is a vector of self-adjoint controller variables. The noise $v_{K}(t)=\left[v_{K 1}(t) \ldots v_{K K_{v}}(t)\right]^{T}$ is a vector of noncommutative Wiener processes with canonical Hermitian Itô matrix $F_{v_{K}}$.

By identifying $\beta_{u}=C_{K} \xi(t)$ and interconnecting (35) and (37), we can obtain the resulting closed-loop system (see [57]). The goal of the $H^{\infty}$ controller synthesis problem is to find a controller (37) for a given disturbance attenuation parameter $g>0$ such that the resulting closed-loop system satisfies (for details, see [57])

$$
\int_{0}^{t}\left\langle\beta_{z}(s)^{T} \beta_{z}(s)+\epsilon \eta(s)^{T} \eta(s)\right\rangle d s \leq\left(g^{2}-\epsilon\right) \int_{0}^{t}\left\langle\beta_{\omega}(s)^{T} \beta_{\omega}(s)\right\rangle d s+\mu_{1}+\mu_{2} t, \forall t>0
$$

for some real constants $\epsilon, \mu_{1}, \mu_{2}>0$, where $\beta_{z}(t)=\left[\begin{array}{ll}C_{1} & D_{12} C_{K}\end{array}\right]\left[\begin{array}{ll}x(t)^{T} & \xi(t)^{T}\end{array}\right]^{T}$.

Furthermore, we suppose that the system (35) satisfies the following assumption (Assumption A): 1) $D_{12}^{T} D_{12}=E_{1}>0$; 2) $\left.D_{21} D_{21}^{T}=E_{2}>0 ; 3\right)$ The matrix $\left[\begin{array}{cc}A-i \omega I & B_{2} \\ C_{1} & D_{12}\end{array}\right]$ is full rank for all $\omega \geq 0 ; 4)$ The matrix $\left[\begin{array}{cc}A-i \omega I & B_{1} \\ C_{2} & D_{21}\end{array}\right]$ is full rank for all $\omega \geq 0$.

The results on quantum $H^{\infty}$ control will be stated in terms of the following pair of algebraic Riccati equations:

$$
\begin{aligned}
& \quad\left(A-B_{2} E_{1}^{-1} D_{12}^{T} C_{1}\right)^{T} X+X\left(A-B_{2} E_{1}^{-1} D_{12}^{T} C_{1}\right)+X\left(B_{1} B_{1}^{T}-g^{2} B_{2} E_{1}^{-1} B_{2}^{T}\right) X \\
& \quad+g^{-2} C_{1}^{T}\left(I-D_{12} E_{1}^{-1} D_{12}^{T}\right) C_{1}=0 \\
& \left(A-B_{1} D_{21}^{T} E_{2}^{-1} C_{2}\right) Y+Y\left(A-B_{1} D_{21}^{T} E_{2}^{-1} C_{2}\right)^{T}+Y\left(g^{-2} C_{1}^{T} C_{1}-C_{2}^{T} E_{2}^{-1} C_{2}\right) Y \\
& +B_{1}\left(I-D_{21}^{T} E_{2}^{-1} D_{21}\right) B_{1}^{T}=0
\end{aligned}
$$

where $X$ and $Y$ are positive definite symmetric matrices. The solutions to these Riccati equations will be required to satisfy the following assumption (Assumption B): 1) $A-$ $B_{2} E_{1}^{-1} D_{12}^{T} C_{1}+\left(B_{1} B_{1}^{T}-g^{2} B_{2} E_{1}^{-1} B_{2}^{T}\right) X$ is a stability matrix; 2) $A-B_{1} D_{21}^{T} E_{2}^{-1} C_{2}+$ $Y\left(g^{-2} C_{1}^{T} C_{1}-C_{2}^{T} E_{2}^{-1} C_{2}\right)$ is a stability matrix; 3$)$ The matrix $X Y$ has a spectral radius strictly less than one.

If the Riccati equations (39) and (40) have solutions satisfying Assumption B, a controller of the form (37) will solve the $H^{\infty}$ control problem under consideration and the system matrices of the controller can be constructed from the Riccati solutions as follows:

$$
\begin{aligned}
& A_{K}=A+B_{2} C_{K}-B_{K} C_{2}+\left(B_{1}-B_{K} D_{21}\right) B_{1}^{T} X \\
& B_{K}=(I-Y X)^{-1}\left(Y C_{2}^{T}+B_{1} D_{21}^{T}\right) E_{2}^{-1} \\
& C_{K}=-E_{1}^{-1}\left(g^{2} B_{2}^{T} X+D_{12}^{T} C_{1}\right) .
\end{aligned}
$$

The necessary and sufficient conditions on $H^{\infty}$ controller synthesis can be described as follows:

Theorem 12 Necessity. Consider the system (35) and suppose that Assumption A is satisfied. If there exists a controller of the form (37) such that the resulting closedloop system is strictly bounded real with disturbance attenuation $g$, then the Riccati 
equations (39) and (40) will have stabilizing solutions $X \geq 0$ and $Y \geq 0$ satisfying Assumption B.

Sufficiency. Suppose the Riccati equations (39) and (40) have stabilizing solutions $X \geq 0$ and $Y \geq 0$ satisfying Assumption B. If the controller (37) is such that the matrices $A_{K}, B_{K}, C_{K}$ are as defined in (41), then the resulting closed-loop system will be strictly bounded real with disturbance attenuation $g$.

It is worth noting that when the controller which is constructed via this approach is a quantum controller, we need to take into account the physical realizability conditions using Theorem 11 .

\section{Conclusions and perspectives}

In this paper, we have surveyed developments in quantum control theory and its applications from a control systems perspective. Some results on controllability of quantum systems, open-loop control strategies and closed-loop design methods have been presented. Also, some applications of quantum control theory are briefly mentioned in this survey. Although great progress has been made in this area, quantum control is still in its infancy. Moreover, there exist several essential differences between quantum control theory and classical control theory. For example, controlling quantum entanglement and protecting quantum coherence are important two classes of quantum control tasks. However, no corresponding tasks exist in classical control theory. In classical feedback control, measurement is taken as the main tool of acquiring feedback information to deal with uncertainties and it is assumed that measurement does not affect the measured system. However, measurement in quantum feedback control unavoidably introduces essential uncertainties when we acquire feedback information through measurement to deal with uncertainties of quantum control systems. Hence, it is necessary to develop more new theory and approaches to control quantum phenomena. We now outline several important topics that are worth exploring, emphasizing different aspects of quantum control (a more detailed discussion on mathematical and algorithmic challenges in quantum control can be found in [170]).

(1) Quantum incoherent control. Quantum coherent control is a powerful control paradigm in open-loop quantum control and many important results have been achieved. However, it may not be practical to accomplish some quantum control tasks using only coherent control approaches. Recently, incoherent resources have been proven useful for accomplishing some quantum control tasks. An example of this is the fact that quantum measurement can serve as a useful control tool. However, there are only few results on the use of quantum incoherent control. Hence, it is desirable to develop systematic and new approaches to construct quantum incoherent (or coherent/incoherent hybrid) controllers which are easily physically realized [54].

(2) Quantum feedback control. Feedback plays a key role in classical control theory. It is desirable to develop systematic feedback control methods for emerging quantum engineering applications. Although many results on quantum feedback control have been presented, they are usually constrained to some special cases (e.g., linear feedback, 
Markovian approximation, simple quantum systems). Many fundamental problems still need to be considered: How does the weak measurement [171] affect quantum systems in quantum feedback control? How does one design feedback controllers for nonlinear quantum systems [172] and is it possible to control nonlinear dynamics of quantum systems using linear controllers? What approaches are effective for control of quantum systems with non-Markovian dynamics [156], [157], [173]? What is the capability of feedback to deal with uncertainties in quantum systems? How do we synthesize complex quantum feedback network systems [174?

(3) Robust control of quantum systems. It is inevitable that quantum systems are subject to many kinds of uncertainties (including disturbances and noises). Hence, the requirement of robustness in the presence of uncertainties has been recognized as one of the key tasks for developing practical quantum technologies. Existing results in this area focus mainly on quantum systems whose dynamics can be described by linear equations in the Heisenberg picture [57], [175] or whose uncertainties can be approximately described as a perturbation in the system Hamiltonian. It is desirable to develop systematic robust control approaches to deal with more general kinds of uncertainties existing in practical quantum systems.

(4) Decoherence control. Decoherence is the main obstacle to the application of quantum information technology. Many quantum control systems are essentially open quantum systems [29, [176] and interaction with external environments (including external controls and the measurement apparatus) unavoidably introduces decoherence [177]. A typical example of decoherence control is quantum error correction [66], [67, [154. Several decoherence control approaches have been proposed. For example, "bang-bang" control has been proposed for dynamic decoherence suppression in two-state quantum systems [111. A classical feedback strategy has been proposed to eliminate decoherence in open quantum systems [178]. Optimal control techniques have been applied to decoherence control of Markovian and non-Markovian open quantum systems [173], [179], [180]. In spite of these efforts, there are still many challenges for decoherence control and the development of systematic and practical decoherence control methods is still an important problem.

(5) Entanglement control. Quantum entanglement is the most important resource in quantum information technology. Several optimal control and feedback control approaches have been proposed to control quantum entanglement [61, [69], 131], 132], [133, 181], [182]. However, existing results are only a first step in entanglement control. Quantum entanglement has many unique characteristics which have no classical counterparts. Hence, it is desirable to develop completely new methods to control quantum entanglement. Moreover, we still lack a suitable theory that can effectively characterize quantum entanglement. This makes the development of a control theory for quantum entanglement more challenging. 


\section{Acknowledgment}

The authors thank the editor James Lam for inviting them to write this survey. The authors would like to thank the referees and K Jacobs, M R James, A I Maalouf, H I Nurdin, A Pechen, B Qi, H Rabitz, S Z Sayed Hassen, T J Tarn, I Vladimirov, R B $\mathrm{Wu}, \mathrm{M}$ Yanagisawa and G F Zhang for their helpful comments and suggestions. The authors also thank D Burgarth, V Giovannetti and F Nori for pointing out several useful references.

\section{References}

[1] Butkovskii, A.G., and Samoilenko, Yu.I.: 'Control of quantum systems', Automat. Rem. Control, 1979, 40, pp.485-502.

[2] Butkovskii, A.G., and Samoilenko, Yu.I.: 'Control of quantum systems (II)', Automat. Rem. Control, 1979, 40, pp.629-645.

[3] Huang, G.M., Tarn, T.J., and Clark, J.W.: 'On the controllability of quantummechanical systems', J. Math. Phys., 1983, 24, pp.2608-2618.

[4] Belavkin, V.P.: 'On the theory of control of observable quantum systems', Automat. Rem. Control, 1983, 44, pp.178-188.

[5] Warren, W.S., Rabitz, H., and Dahleh, M.: 'Coherent control of quantum dynamics: The dream is alive', Science, 1993, 259, pp.1581-1589.

[6] Chu, S.: 'Cold atoms and quantum control', Nature, 2002, 416, pp.206-210.

[7] Mabuchi, H., and Khaneja, N.: 'Principles and applications of control in quantum systems', Int. J. Robust Nonlinear Control, 2005, 15, pp.647-667.

[8] Rabitz, H.: 'Focus on quantum control' New J. Phys., 2009, 11, p.105030.

[9] Rabitz, H., de Vivie-Riedle, R., Motzkus, M., and Kompa, K.: 'Whither the future of controlling quantum phenomena?' Science, 2000, 288, pp.824-828.

[10] Rabitz, H.: 'The role of theory in the laboratory control of quantum dynamics phenomena', Theor. Chem. Acc., 2003, 109, pp.64-70.

[11] Rice, S.A., and Zhao, M.S.: Optical Control of Molecular Dynamics, (John Wiley \& Sons, Inc., 2000, 1st edn).

[12] Shapiro, M., and Brumer, P.: Principles of the Quantum Control of Molecular Processes, (John Wiley \& Sons, Inc., 2003, 1st edn).

[13] Dantus, M., and Lozovoy, V.V.: 'Experimental coherent laser control of physicochemical processes', Chem. Rev., 2004, 104, pp.1813-1859. 
[14] Bonačić-Koutecký, V., and Mitrić, R.: 'Theoretical exploration of ultrafast dynamics in atomic clusters: analysis and control', Chem. Rev., 2005, 105, pp.11-65.

[15] Wiseman, H.M., and Milburn, G.J.: 'Quantum theory of optical feedback via homodyne detection', Phys. Rev. Lett., 1993, 70, pp.548-551.

[16] van Handel, R., Stockton, J.K., and Mabuchi, H.: 'Modelling and feedback control design for quantum state preparation', J. Opt. B: Quantum Semiclass. Opt., 2005, 7, pp.S179-S197.

[17] Lloyd, S.: 'Coherent quantum feedback', Phys. Rev. A, 2000, 62, p.022108.

[18] Dowling, J.P., and Milburn, G.J.: 'Quantum technology: the second quantum revolution', Phil. Trans. R. Soc. Lond. A, 2003, 361, pp.1655-1674.

[19] Ramakrishna, V., and Rabitz, H.: 'Relation between quantum computing and quantum controllability', Phys. Rev. A, 1996, 54, pp.1715-1716.

[20] Ramakrishna, V., Salapaka, M.V., Dahleh, M., Rabitz, H., and Peirce, A.: 'Controllability of molecular systems', Phys. Rev. A, 1995, 51, pp.960-966.

[21] Wu, R.B., Tarn, T.J., and Li, C.W.: 'Smooth controllability of infinitedimensional quantum-mechanical systems', Phys. Rev. A, 2006, 73, p.012719.

[22] Schirmer, S.G., Fu, H., and Solomon, A.I.: 'Complete controllability of quantum systems', Phys. Rev. A, 2001, 63, p.063410.

[23] Zhang, C.B., Dong, D.Y., and Chen, Z.H.: 'Control of non-controllable quantum systems: a quantum control algorithm based on Grover iteration,' J. Opt. B: Quantum Semiclass. Opt., 2005, 7, pp.S313-S317.

[24] Albertini, F., and D'Alessandro, D.: 'Notions of controllability for bilinear multilevel quantum systems', IEEE Trans. Autom. Control, 2003, 48, pp.1399-1403.

[25] D'Alessandro, D.: Introduction to Quantum Control and Dynamics, (Chapman \& Hall/CRC, 2007, 1st edn.)

[26] Wu, R., Pechen, A., Brif, C., and Rabitz, H.: 'Controllability of open quantum systems with Kraus-map dynamics', J. Phys. A: Math. Theor., 2007, 40, pp.56815693.

[27] Turinici, G., and Rabitz, H.: 'Quantum wavefunction controllability', Chem. Phys., 2001, 267, pp.1-9.

[28] Turinici, G., and Rabitz, H.: 'Wavefunction controllability for finite-dimensional bilinear quantum systems', J. Phys. A: Math. Gen., 2003, 36, pp.2565-2576.

[29] Breuer, H.-P., and Petruccione, F.: The Theory of Open Quantum Systems, (Oxford University Press, 2002, 1st edn). 
[30] Altafini, C.: 'Controllability properties for finite dimensional quantum Markovian master equations', J. Math. Phys., 2003, 44, pp.2357-2372.

[31] Rice, S.A.: 'Interfering for the good of a chemical reaction', Nature, 2001, 409, pp.422-426.

[32] Shapiro, M., and Brumer, P.: 'Quantum control of bound and continuum state dynamics', Phys. Rep., 2006, 425, pp.195-264.

[33] Peirce, A.P., Dahleh, M., and Rabitz, H.: 'Optimal control of quantummechanical systems: Existence, numerical approximation, and applications', Phys. Rev. A, 1988, 37, pp.4950-4964.

[34] Dahleh, M., Peirce, A.P., and Rabitz, H.: 'Optimal control of uncertain quantum systems', Phys. Rev. A, 1990, 42, pp.1065-1079.

[35] Khaneja, N., Brockett, R., and Glaser, S.J.: 'Time optimal control in spin systems', Phys. Rev. A, 2001, 63, p.032308.

[36] Boscain, U., Charlot, G., Gauthier, J.P., Guérin, S., and Jauslin, H.R.: 'Optimal control in laser-induced population transfer for two- and three-level quantum systems', J. Math. Phys., 2002, 43, pp.2107-2132.

[37] Boscain, U., and Mason, P.: 'Time minimal trajectories for a spin 1/2 particle in a magnetic field', J. Math. Phys., 2006, 47, p.062101.

[38] Khaneja, N., Luy, B., and Glaser, S.J.: 'Boundary of quantum evolution under decoherence,' Proc. Natl. Acad. Sci. USA, 2003, 100, pp.13162-13166.

[39] Khaneja, N., Reiss, T., Luy, B., and Glaser, S.J.: 'Optimal control of spin dynamics in the presence of relaxation', J. Magn. Reson., 2003, 162, pp.311-319.

[40] Khaneja, N., Kehlet, C., Luy, B., and Glaser, S.J.: 'Broadband relaxation optimized polarization transfer in magnetic resonance,' Proc. Natl. Acad. Sci. USA, 2003, 101, pp.14742-14747.

[41] Mirrahimi, M., Rouchon, P., and Turinici, G.: 'Lyapunov control of bilinear Schrödinger equations', Automatica, 2005, 41, pp.1987-1994.

[42] Kuang, S., and Cong, S.: 'Lyapunov control methods of closed quantum systems', Automatica, 2008, 44, pp.98-108.

[43] Dong, D., and Petersen, I.R.: 'Variable structure control of uncontrollable quantum systems', Proc. 6th IFAC Symposium Robust Control Design, p.16, June 2009, Haifa, Israel.

[44] Dong, D., and Petersen, I.R.: 'Controllability of quantum systems with switching control', Int. J. Control, 2010, doi:10.1080/00207179.2010.538437. 
[45] Vilela Mendes, R., and Man'ko, V.I.: 'Quantum control and the Strocchi map', Phys. Rev. A, 2003, 67, p.053404.

[46] Shuang, F., Pechen, A., Ho, T.S., and Rabitz, H.: 'Observation-assisted optimal control of quantum dynamics', J. Chem. Phys., 2007, 126, p.134303.

[47] Pechen, A., Il'in, N., Shuang, F., and Rabitz, H.: 'Quantum control by von Neumann measurements', Phys. Rev. A, 2006, 74, p.052102.

[48] Mandilara, A., and Clark, J.W.: 'Probabilistic quantum control via indirect measurement', Phys. Rev. A, 2005, 71, p.013406.

[49] Romano, R., and D'Alessandro, D.: 'Environment-mediated control of a quantum system', Phys. Rev. Lett., 2006, 97, p.080402.

[50] Romano, R., and D'Alessandro, D.: 'Incoherent control and entanglement for two-dimensional coupled Systems', Phys. Rev. A, 2006, 73, p.022323.

[51] Dong, D., Chen, C., Tarn, T.J., Pechen, A., and Rabitz, H.: 'Incoherent control of quantum systems with wavefunction controllable subspaces via quantum reinforcement learning', IEEE Trans. Syst., Man, Cybern., Part B: Cybern., 2008, 38, pp.957-962.

[52] Dong, D., Lam, J., and Tarn, T.J.: 'Rapid incoherent control of quantum systems based on continuous measurements and reference model', IET Control Theory Appl., 2009, 3, pp.161-169.

[53] Dong, D., Lam, J., and Petersen, I.R.: 'Robust incoherent control of of qubit systems via switching and optimisation', Int. J. Control, 2010, 83, pp.206-217.

[54] Pechen, A., and Rabitz, H.: 'Teaching the environment to control quantum systems', Phys. Rev. A, 2006, 73, p.062102.

[55] Dong, D., Zhang, C., Rabitz, H., Pechen, A., and Tarn, T.J.: 'Incoherent control of locally controllable quantum systems', J. Chem. Phys., 2008, 129, p.154103.

[56] Gardiner, C.W., and Zoller, P.: Quantum Noise, (New York: Springer-Verlag, 2000, 2nd edn).

[57] James, M.R., Nurdin, H.I., and Petersen, I.R.: ' $\mathrm{H}^{\infty}$ control of linear quantum stochastic systems', IEEE Trans. Autom. Control, 2008, 53, pp.1787-1803.

[58] Judson, R.S., and Rabitz, H.: 'Teaching lasers to control molecules', Phys. Rev. Lett., 1992, 68, pp.1500-1503.

[59] Scully, M.O., and Zubairy, M.S.: Quantum Optics, (Cambridge: Cambridge University Press, 1997, 1st edn).

[60] Thomsen, L.K., Mancini, S., and Wiseman, H.M.: 'Spin squeezing via quantum feedback,' Phys. Rev. A, 2002, 65, p.061801(R). 
[61] Yamamoto, N., Tsumura, K., and Hara, S.: 'Feedback control of quantum entanglement in a two-spin system', Automatica, 2007, 43, pp.981-992.

[62] van Handel, R., Stockton, J.K., and Mabuchi, H.: 'Feedback control of quantum state reduction', IEEE Trans. Autom. Control, 2005, 50, pp.768-780.

[63] Mirrahimi, M., and van Handel, R.: 'Stabilizing feedback controls for quantum systems', SIAM J. Control Optim., 2007, 46, pp.445-467.

[64] Jacobs, K.: "How to project qubits faster using quantum feedback", Phys. Rev. A, 2003, 67, p.030301(R).

[65] Combes, J., and Jacobs, K.: 'Rapid state reduction of quantum systems using feedback control', Phys. Rev. Lett., 2006, 96, p.010504.

[66] Ahn, C., Doherty, A.C., and Landahl, A.J.: 'Continuous quantum error correction via quantum feedback control', Phys. Rev. A, 2002, 65, p.042301.

[67] Sarovar, M., Ahn, C., Jacobs, K., and Milburn, G.J.: 'Practical scheme for error control using feedback', Phys. Rev. A, 2004, 69, p.052324.

[68] Jacobs, K., and Shabani, A.: 'Quantum feedback control: How to use verification theorems and viscosity solutions to find optimal protocols', Contemp. Phys., 2008, 49, pp.435-448.

[69] Mancini, S., and Wiseman, H.M.: 'Optimal control of entanglement via quantum feedback', Phys. Rev. A, 2007, 75, p.012330.

[70] Belavkin, V.P.: 'Measurement, filtering and control in quantum open dynamical systems', Rep. Math. Phys., 1999, 43, pp.405-425.

[71] Bouten, L., van Handel, R., and James, M.R.: 'An introduction to quantum filtering', SIAM J. Control Optim., 2007, 46, pp.2199-2241.

[72] Bouten, L., van Handel, R., and James, M.R.: 'A discrete invitation to quantum filtering and feedback control', SIAM Review, 2009, 51, pp.239-316.

[73] Doherty, A.C., and Jacobs, K.: 'Feedback control of quantum systems using continuous state estimation', Phys. Rev. A, 1999, 60, pp.2700-2711.

[74] Geremia, J.M., Stockton, J.K., Doherty, A.C., and Mabuchi, H.: 'Quantum Kalman filtering and the Heisenberg limit in atomic magnetometry', Phys. Rev. Lett., 2003, 91, p.250801.

[75] Stockton, J.K., Geremia, J.M., Doherty, A.C., and Mabuchi, H.: 'Robust quantum parameter estimation: Coherent magnetometry with feedback', Phys. Rev. A, 2004, 69, p.032109. 
[76] Geremia, J.M., and Rabitz, H.: 'Optimal identification of Hamiltonian information by closed-loop laser control of quantum systems', Phys. Rev. Lett., 2002, 89, p.263902.

[77] Bonnabel, S., Mirrahimi, M., and Rouchon, P.: 'Observer-based Hamiltonian identification for quantum systems', Automatica, 2009, 45, pp.1144-1155.

[78] Burgarth, D., Maruyama, K., and Nori, F.: 'Coupling strength estimation for spin chains despite restricted access', Phys. Rev. A, 2009, 79, p.020305(R).

[79] D'Helon, C., and James, M.R.: 'Stability, gain, and robustness in quantum feedback networks,' Phys. Rev. A, 2006, 73, p.053803.

[80] Nielsen, M.A., and Chuang, I.L.:Quantum Computation and Quantum Information, (Cambridge University Press, 2000, 1st edn.)

[81] Vandersypen, L.M.K., and Chuang, I.L.: 'NMR techniques for quantum control and computation', Rev. Mod. Phys., 2004, 76, pp.1037-1069.

[82] Wiseman, H.M., and Milburn, G.J.: Quantum Measurement and Control, (Cambridge University Press, 2010, 1st edn).

[83] Dirac, P.A.M.: The Principles of Quantum Mechanics, (Oxford University Press, 1958, 4th edn).

[84] Landau, L.D., and Lifshitz, E.M.: Quantum Mechanics (Non-relativistic Theory), (Butterworth-Heinemann, 1999, 3rd edn).

[85] Dong, D., Chen, C., Li, H., and Tarn, T.J.: 'Quantum reinforcement learning', IEEE Trans. Syst., Man, Cybern., Part B: Cybern., 2008, 38, pp.1207-1220.

[86] Preskill, J.: Physics 229: Advanced Mathematical Methods of Physics-Quantum Information and Computation, (California Institute of Technology, 1998). Available electronically via http://www.theory.caltech.edu/people/preskill/ph229/

[87] Jacobs, K.: 'Optimal feedback control for rapid preparation of a qubit', Proc. SPIE, 2004, 5468, pp.355-364.

[88] Brun, T.A.: 'A simple mode of quantum trajectories', Am. J. Phys., 2002, 70, pp.719-737.

[89] Jacobs, K., and Steck, D.A.: 'A straightforward introduction to continuous quantum measurement', Contemp. Phys., 2006, 47, pp.279-303.

[90] Korotkov, A.N.: 'Selective quantum evolution of a qubit state due to continuous measurement', Phys. Rev. B, 2001, 63, p.115403.

[91] D'Alessandro, D., and Dahleh, M.: 'Optimal control of two-level quantum systems', IEEE Trans. Autom. Control, 2001, 46, pp.866-876. 
[92] Lindblad, G.: 'On the generators of quantum dynamical semigroups,' Commun. Math. Phys., 1976, 48, pp.119-130.

[93] Alicki, R., and Lendi, K.: Quantum Dynamical Semigroups and Applications, (Springer, 2007, 2nd edn).

[94] Qi, B.: 'On the quantum master equation under feedback control,' Sci. China Ser. F - Inf. Sci., 2009, 52, pp.2133-2139.

[95] Parthasarathy, K.R.: An Introduction to Quantum Stochastic Calculus, (Berlin, Germany, Birkhauser, 1992, 1st edn).

[96] Belavkin, V.P.: 'Quantum continual measurements and a posteriori collapse on CCR,' Commun. Math. Phys., 1992, 146, pp. 611-635.

[97] Schirmer, S.G., Solomon, A.I., and Leahy, J.V.: 'Degrees of controllability for quantum systems and application to atomic systems', J. Phys. A: Math. Gen., 2002, 35, pp.4125-4141.

[98] Altafini, C.: 'Controllability of quantum mechanical systems by root space decomposition of $\mathrm{su}(N)$ ', J. Math. Phys., 2002, 43, pp.2051-2062.

[99] Lan, C.H., Tarn, T.J., Chi, Q.S., and Clark, J.W.: 'Analytic controllability of time-dependent quantum control systems', J. Math. Phys., 2005, 46, p.052102.

[100] Kraus, K.: States, Effects, and Operations, (Springer, 1983, 1st edn).

[101] Lloyd, S., and Viola, L.: 'Engineering quantum dynamics', Phys. Rev. A, 2001, 65, p.010101(R).

[102] Ramakrishna, V., Flores, K.L., Rabitz, H., and Ober, R.J.: 'Quantum control by decompositions of SU(2),' Phys. Rev. A, 2000, 62, p.053409.

[103] Khaneja, N., and Glaser, S.J.: 'Cartan decomposition of $\mathrm{SU}\left(2^{n}\right)$ and control of spin systems,' Chem. Phys., 2001, 267, pp.11-23.

[104] Werschnik, J., and Gross, E.K.U.: 'Quantum optimal control theory', J. Phys. B: At. Mol. Opt. Phys., 2007, 40, pp.R175-R211.

[105] Sugny, D., Kontz, C., and Jauslin, H.R.: 'Time-optimal control of a two-level dissipative quantum system', Phys. Rev. A, 2007, 76, p.023419.

[106] Grivopoulos, S., and Bamieh, B.: 'Optimal population transfers in a quantum system for large transfer time,' IEEE Trans. Autom. Control, 2008, 53, pp.980992.

[107] Zhu, W.S., and Rabitz, H.: 'A rapid monotonically convergent iteration algorithm for quantum optimal control over the expectation value of a positive definite operator', J. Chem. Phys., 1998, 109, pp.385-391. 
[108] Rabitz, H., Hsieh, M.M., and Rosenthal, C.M.: 'Quantum optimally controlled transition landscapes,' Science, 2004, 303, pp.1998-2001.

[109] Chakrabarti, R., and Rabitz, H.: 'Quantum control landscape,' Int. Rev. Phys. Chem., 2007, 26, pp.671-735.

[110] Yuan, H., and Khaneja, N.: 'Time optimal control of coupled qubits under nonstationary interactions', Phys. Rev. A, 2005, 72, p.040301(R).

[111] Viola, L., and Lloyd, S.: 'Dynamical suppression of decoherence in two-state quantum systems', Phys. Rev. A, 1998, 58, pp.2733-2744.

[112] Wu, J.W., Li, C.W., Tarn, T.J. and Zhang, J.: 'Optimal bang-bang control for SU(1,1) coherent states', Phys. Rev. A, 2007, 76, p.053403.

[113] D'Alessandro, D.: 'Optimal evaluation of generalized Euler angles with applications to control', Automatica, 2004, 40, pp.1997-2002.

[114] Chen, B.S., Chen, W.H., Hsu, F., and Zhang, W.: 'Optimal tracking control design of quantum systems via tensor formal power series method,' Open Autom. Control Syst. J., 2008, 1, pp.50-64.

[115] Ferrante, A., Pavon, M., and Raccanelli, G.: 'Driving the propagator of a spin system: a feedback approach', Proc. of the 41st IEEE Conf. on Decision and Control, 2002, pp.46-50, Las Vegas, Nevada USA.

[116] Vettori, P.: 'On the convergence of a feedback control strategy for multilevel quantum systems', Proc. the Fifteenth Int. Symposium MTNS, South Bend, Indiana, USA, 2002.

[117] Grivopoulos, S., and Bamieh, B.: 'Lyapunov-based control of quantum systems', Proc. of the 42nd IEEE Conf. on Decision and Control, 2003, pp.434-438, Maui, Hawaii USA.

[118] Mirrahimi, M., Turinici, G., and Rouchon, P.: 'Reference trajectory tracking for locally designed coherent quantum controls', J. Phys. Chem. A, 2005, 109, pp.2631-2637.

[119] Beauchard, K., Coron, J.M., Mirrahimi, M., and Rouchon, P.: 'Implicit Lyapunov control of finite dimensional Schrödinger equations,' Syst. Control Lett., 2007, 56, pp.388-395.

[120] Altafini, C.: 'Feedback control of spin systems', Quantum Inform. Process., 2007, 6, pp.9-36.

[121] Altafini, C.: 'Feedback stabilization of isospectral control systems on complex flag manifolds: application to quantum ensembles', IEEE Trans. Autom. Control, 2007, 52, pp.2019-2028. 
[122] Wang, X., and Schirmer, S.G.: 'Analysis of Lyapunov method for control of quantum states', IEEE Trans. Autom. Control, 2010, 55, pp.2259-2270.

[123] Dong, D., and Petersen, I.R.: 'Sliding mode control of quantum systems', New J. Phys., 2009, 11, p.105033.

[124] Altafini, C.: 'Coherent control of open quantum dynamical systems', Phys. Rev. $A, 2004, \mathbf{7 0}$, p.062321.

[125] Roa, L., Delgado, A., Ladrón de Guevara, M.L., and Klimov, A.B.: 'Measurement-driven quantum evolution', Phys. Rev. A, 2006, 73, p.012322.

[126] Gong, J.B., and Rice, S.A.: 'Measurement-assisted coherent control,' J. Chem. Phys., 2004, 120, pp.9984-9988.

[127] Zhang, M., Dai, H.Y., Xi, Z.R., Xie, H.W., and Hu, D.W.: 'Combating dephasing decoherence by periodically performing tracking control and projective measurement', Phys. Rev. A, 2007, 76, p.042335.

[128] Burgarth, D, and Giovannetti, V.: 'Full control by locally induced relaxation', Phys. Rev. Lett., 2007, 99, p.100501.

[129] Burgarth, D., Bose, S., Bruder, C., and Giovannetti, V.: 'Local controllability of quantum networks', Phys. Rev. A, 2009, 79, p.060305(R).

[130] Haus, H.A., and Yamamoto, Y.: 'Theory of feedback-generated states', Phys. Rev. A, 1986, 34, pp.270-292.

[131] Yanagisawa, M.: 'Quantum feedback control for deterministic entangled photon generation,' Phys. Rev. Lett., 2006, 97, p.190201.

[132] Yamamoto, N, Nurdin, H.I., James, M.R., and Petersen, I.R.: 'Avoiding entanglement sudden death via measurement feedback control in a quantum network,' Phys. Rev. A, 2008, 78, p.042339.

[133] Hill, C., and Ralph, J.: 'Weak measurement and control of entanglement generation', Phys. Rev. A, 2008, 77, p.014305.

[134] Wiseman, H.M., and Ralph, J.F.: 'Reconsidering rapid qubit purification by feedback', New J. Phys., 2006, 8, p.90.

[135] Combes, J., Wiseman, H.M., and Jacobs, K.: 'Rapid measurement of quantum systems using feedback control,' Phys. Rev. Lett., 2008, 100, p.160503.

[136] Jordan, A.N., and Korotkov, A.N.: 'Qubit feedback and control with kicked quantum nondemolition measurements: A quantum Bayesian analysis', Phys. Rev. B, 2008, 74, p.085307.

[137] Wiseman, H.M., and Bouten, L.: 'Optimality of feedback control strategies for qubit purification,' Quantum Inform. Proc., 2008, 7, pp.71-83. 
[138] Griffith, E.J., Hill, C.D., Ralph, J.F., Wiseman, H.M., and Jacobs, K.: 'Rapidstate purification protocols for a Cooper pair box', Phys. Rev. B, 2007, 75, p.014511.

[139] Wilson, S.D., Carvalho, A.R.R., Hope, J.J., and James, M.R.: 'Effects of measurement backaction in the stabilization of a Bose-Einstein condensate through feedback', Phys. Rev. A, 2007, 76, p.013610.

[140] Zhang, J., Liu, Y.X., and Nori, F.: 'Cooling and squeezing the fluctuations of a nanomechanical beam by indirect quantum feedback control', Phys. Rev. A, 2009, 79, p.052102.

[141] Qi, B., and Guo, L.: 'Is measurement-based feedback still better for quantum control systems?' Syst. Control Lett., 2010, 59, pp.333-339.

[142] Wiseman, H.M.: 'Quantum theory of continuous feedback'' Phys. Rev. A, 1994, 49, pp.2133-2150.

[143] Doherty, A.C., Habib, S., Jacobs, K., Mabuchi, H., and Tan, S.M.: 'Quantum feedback control and classical control theory,' Phys. Rev. A, 2000, 62, p.012105.

[144] Doherty, A.C., Jacobs, K., and Jungman, G.: 'Information, disturbance, and Hamiltonian quantum feedback control,' Phys. Rev. A, 2001, 63, p.062306.

[145] Wiseman, H.M., Mancini, S., and Wang, J.: 'Bayesian feedback versus Markovian feedback in a two-level atom,' Phys. Rev. A, 66, p.013807.

[146] Braginsky, V.B., and Khalili, F.Ya.: 'Quantum nondemolition measurements: the route from toys to tools', Rev. Mod. Phys., 1996, 68, pp.1-11.

[147] Mabuchi, H.: 'Dynamical identification of quantum open quantum systems', Quantum Semiclass. Opt., 1996, 8, pp.1103-1108.

[148] Gambetta, J., and Wiseman, H.M.: 'State and dynamical parameter estimation for open quantum systems,' Phys. Rev. A, 2001, 64, p.042105.

[149] James, M.R.: 'Risk-sensitive optimal control of quantum systems', Phys. Rev. A, 2004, 69, p.032108.

[150] Yamamoto, N.: 'Robust observer for uncertain linear quantum systems', Phys. Rev. A, 2006, 74, p.032107.

[151] Yamamoto, N., and Bouten, L.: 'Quantum risk-sensitive estimation and robustness', IEEE Trans. Autom. Control, 2009, 54, pp.92-107.

[152] Wang, J., and Wiseman, H.M.: 'Feedback-stabilization of an arbitrary pure state of a two-level atom,' Phys, Rev. A, 2001, 64, p.063810.

[153] Ticozzi, F., and Viola, L.: 'Quantum Markovian subsystems: invariance, attractivity, and control,' IEEE Trans. Autom. Control, 2008, 53, pp.2048-2063. 
[154] Ahn, C., Wiseman, H.M., Milburn, G.J.: 'Quantum error correction for continuously detected errors,' Phys. Rev. A, 2003, 67, p.052310.

[155] Ruskov, R., and Korotkov, A.N.: 'Quantum feedback control of a solid-state qubit,' Phys. Rev. B, 66, p.041401(R).

[156] Giovannetti, V., Tombesi, P., and Vitali, D.: 'Non-Markovian quantum feedback from homodyne measurements: The effect of a nonzero feedback delay time', Phys. Rev. A, 1999, 60, pp.1549-1561.

[157] Wang, J., Wiseman, H.M., and Milburn, G.J.: 'Non-Markovian homodynemediated feedback on a two-level atom: a quantum trajectory treatment', Chem. Phys., 2001, 268, pp.221-235.

[158] Mabuchi, H.: 'Coherent-feedback quantum control with a dynamic compensator,' Phys. Rev. A, 2008, 78, p.032323.

[159] Nurdin, H.I., James, M.R., and Petersen, I.R.: 'Coherent quantum LQG control', Automatica, 2009, 45, pp.1837-1846.

[160] Wiseman, H.M., and Doherty, A.C.: 'Optimal unravellings for feedback control in linear quantum systems', Phys. Rev. Lett., 2005, 94, p.070405.

[161] Zhang, Z.: 'Effect of input noise on a magnetometer with quantum feedback,' SIAM J. Control Optim., 2008, 47, pp. 639-660.

[162] Shaiju, A.J., Petersen, I.R., and James, M.R.: 'Guaranteed cost LQG control of uncertain linear quantum stochastic systems,' Proc. of the 2007 American Control Conf., 2007, pp.2118-2123, New York City, USA.

[163] Sayed Hassen, S.Z., Heurs, M., Huntington, E.H., Petersen, I.R., and James, M.R.: 'Frequency locking of an optical cavity using linear-quadratic Gaussian integral control,' J. Phys. B: At. Mol. Opt. Phys., 2009, 42, p. 175501.

[164] Nurdin, H.I., James, M.R., and Doherty, A.C.: 'Network synthesis of linear dynamical quantum stochastic systems,' SIAM J. Control Optim., 2009, 48, pp.2686-2718.

[165] Zhang, H., and Rabitz, H.: 'Robust optimal control of quantum molecular systems in the presence of disturbances and uncertainties,' Phys. Rev. A, 1994, 49, pp.2241-2254.

[166] Bacon, D., Lidar, D.A., Whaley, K.B.: 'Robustness of decoherence-free subspaces for quantum computation', Phys. Rev. A, 1999, 60, pp.1944-1955.

[167] Rabitz, H.: 'Optimal control of quantum systems: Origins of inherent robustness to control field fluctuations,' Phys. Rev. A, 2002, 66, p.063405. 
[168] Yanagisawa, M., and Kimura, H.: 'Transfer function approach to quantum control-part I: Dynamics of quantum feedback systems', IEEE Trans. Autom. Control, 2003, 48, pp.2107-2120.

[169] Yanagisawa, M., and Kimura, H.: 'Transfer function approach to quantum control-part II: Control concepts and applications', IEEE Trans. Autom. Control, 2003, 48, pp.2121-2132.

[170] Brown, E., and Rabitz, H.: 'Some mathematical and algorithmic challenges in the control of quantum dynamics phenomena,' J. Math. Chem., 2002, 31, pp.17-63.

[171] Lloyd, S., and Slotine, J.J.E.: 'Quantum feedback with weak measurements,' Phys. Rev. A, 2000, 62, p.012307.

[172] Jacobs, K., and Lund, A.P.: 'Feedback control of nonlinear quantum systems: a rule of thumb,' Phys. Rev. Lett., 2007, 99, p.020501.

[173] Cui, W., Xi, Z.R., and Pan, Y.: 'Optimal decoherence control in non-Markovian open dissipative quantum systems', Phys. Rev. A, 2008, 77, p.032117.

[174] Gough, J. and James, M.R.: 'The series product and its application to quantum feedforward and feedback networks,' IEEE Trans. Autom. Control, 2009, 54, pp. $2530-2544$.

[175] Maalouf, A.I., and Petersen, I.R.: 'Coherent $H^{\infty}$ control for a class of linear complex quantum systems,' Proc. of the 2009 American Control Conf., 2009, pp.1472-1479, St. Louis, MO, USA.

[176] Carmichael, H.J.: An Open Systems Approach to Quantum Optics, (SpringerVerlag, Berlin Heidelberg New-York, 1993, 1st edn).

[177] Zhang, J., Wu, R.B., Li, C.W., Tarn, T.J., and Wu, J.W.: 'Asymptotically noise decoupling for Markovian open quantum systems', Phys. Rev. A, 2007, 75, p.022324.

[178] Ganesan, N., and Tarn, T.J.: 'Decoherence control in open quantum systems via classical feedback', Phys. Rev. A, 2007, 75, p.032323.

[179] Zhang, J., Li, C.W., Wu, R.B., Tarn, T.J., and Liu, X.S.: 'Maximal suppression of decoherence in Markovian quantum systems', J. Phys. A: Math. Gen., 2005, 38, pp.6587-6601.

[180] Gordon, G., and Kurizki, G.: 'Optimal dynamical decoherence control of a qubit', Phys. Rev. Lett., 2008, 101, p.010403.

[181] Zhang, J., Wu, R.B., Li, C.W. and Tarn, T.J.: 'Protecting coherence and entanglement by quantum feedback controls,' IEEE Trans. Autom. Control, 2010, 55, pp.619-633. 
[182] Cui, W., Xi, Z.R., and Pan, Y.: 'Non-Markovian entanglement dynamic between two oscillators in the same environment', J. Phys. A: Math. Theor., 2009, 42, p.155303. 